\title{
Measurements and modeling of plasma flow damping in the Helically Symmetric eXperiment ${ }^{a)}$
}

\author{
S. P. Gerhardt, ${ }^{\text {b) }}$ J. N. Talmadge, J. M. Canik, and D. T. Anderson \\ HSX Plasma Laboratory, University of Wisconsin-Madison, Madison, Wisconsin 53706
}

(Received 15 November 2004; accepted 26 January 2005; published online 22 April 2005)

\begin{abstract}
Measurements of plasma flow damping have been made in the Helically Symmetric eXperiment [F. S. B. Anderson, A. F. Almagri, D. T. Anderson, P. G. Mathews, J. N. Talmadge, and J. L. Shohet, Fusion Technology 27, 273 (1995)] using a biased electrode to impulsively spin the plasma and Mach probes to measure the rotation. There is a distinct asymmetry between the spin-up when the bias is initiated and relaxation when the electrode current is broken. In each case, two time-scales are observed in the evolution of the plasma flow. These observations motivate the development of new neoclassical modeling techniques, including a new model where the fast increment of the electric field initiates the spin-up process. The flow in the quasisymmetric configuration rises more slowly and to a higher value than in a configuration with the quasisymmetry broken, and the rise time-scale is in reasonable agreement with the neoclassical spin-up model. The flows decay more slowly in the quasisymmetry configuration than in the configuration with the quasisymmetry broken, although the decay rates are significantly faster than the neoclassical prediction. () 2005 American Institute of Physics. [DOI: 10.1063/1.1876293]
\end{abstract}

\section{INTRODUCTION}

A new generation of advanced stellarator devices has recently been studied based on the concept of quasisymmetry. ${ }^{1,2}$ These devices have the property that the Fourier spectrum of $|B|$ on a magnetic surface is dominated by a single harmonic. These stellarators possess neoclassical transport at or beneath the level of an axisymmetric system, as well as the inherently steady state properties which all members of the stellarator family share.

There are many advantages of quasisymmetric stellarators compared to their conventional cousins. Conventional stellarators have a class of particle orbits which leave the confinement volume without collisions; quasisymmetric stellarators are able to eliminate the majority of these "direct loss" orbits. In the low collisionality regime, the transport coefficients in a traditional stellarator can scale like $1 / \nu$, where $\nu$ is the collision frequency. Quasisymmetric stellarators are largely able to suppress this transport scaling in favor of the conventional bananna regime scaling $(D \propto \nu)$.

A further advantage of quasisymmetric stellarators lies in their reduced neoclassical damping of plasma flows. Conventional stellarators have large viscous damping of flows in all directions on a magnetic surface because of the complicated variation of $|B|$ on the surface. A quasisymmetric stellarator possesses a direction of symmetry in $|B|$ and the neoclassical viscous damping of flows in this direction is minimal. The ability to sustain plasma flows may be an important ingredient in developing improved confinement regimes, ${ }^{3}$ and has been invoked as a potential advantage in the designs of the quasitoroidally symmetric National Com-

\footnotetext{
a) Paper EI1 3, Bull. Am. Phys. Soc. 49, 98 (2004).

${ }^{b}$ Invited speaker. Present address: Princeton Plasma Physics Laboratory, Princeton, NJ 08543.
}

pact Stellarator eXperiment ${ }^{4}$ (NCSX) and compact helical system, quasiaxisymmetric (CHS-qa), ${ }^{5}$ and the quasipoloidally symmetric stellarator. ${ }^{6}$ It is the purpose of this paper to present measurements and modeling of biased plasma flows in the quasihelically symmetric Helically Symmetric eXperiment (HSX), ${ }^{7}$ which demonstrate the reduction of plasma flow damping with quasisymmetry. ${ }^{8}$

The subject of flow damping in stellarators has received limited experimental attention. Measurements in CHS have shown that in configurations with large toroidal viscosity, the damping of neutral beam induced toroidal flows can be described by neoclassical theory. ${ }^{9}$ In configurations of the device where the toroidal viscosity is reduced, anomalous shear viscosity is necessary to explain the measured damping. A similar result was observed in Wendelstein-7 Advanced Stellarator (W7-AS). ${ }^{10}$ Experiments in the small stellarator IMS found good agreement of the measured radial conductivity and flow decay times ${ }^{11}$ with the neoclassical model of Coronado and Talmadge. ${ }^{12}$

The unique feature of the HSX device is the quasihelically symmetric magnetic field. This magnetic field is created by a set of 48 nonplanar modular coils, with eight each of six different types of coils. We describe the magnetic field produced by these coils by the Fourier decomposition of $|B|$ on a magnetic surface:

$$
B=B_{0} \sum_{\mathrm{n}, \mathrm{m}} b_{\mathrm{nm}} \cos (n \zeta-m \alpha) .
$$

In this expression, $\alpha$ is the poloidal angle and $\zeta$ is the toroidal angle. In a quasisymmetric system, this sum will be dominated by a single term (or terms of all the same helicity). This is illustrated in Fig. 1(a), where the magnetic field spectrum is shown for the quasihelically symmetric (QHS) configuration of HSX (the angles in this figure are the Ha- 


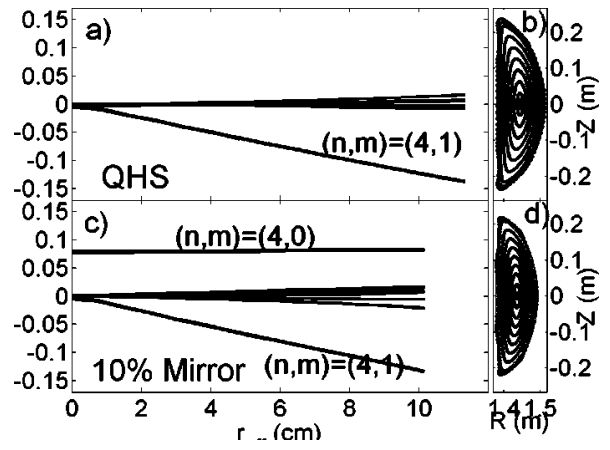

FIG. 1. The Fourier decomposition of the magnetic field in Hamada coordinates for (a) the QHS and (c) mirror configurations of HSX. The magnetic surfaces in a vertical cut at the elliptical symmetry plane are also shown for (b) the QHS and (d) the mirror configurations.

mada angles, see Sec. IV). The $|B|$ spectrum is dominated by a single spectral component with toroidal $(n)$ and poloidal ( $m$ ) mode numbers $(n, m)=(4,1)$. A traditional stellarator would have an $(n, m)=(0,-1)$ spectral component, whose amplitude is equal to the inverse aspect ratio of the magnetic surface under consideration. In HSX, this spectral component has been suppressed to the extent that an aspect ratio of $\sim 400$ would be inferred from the amplitude of the $(n, m)$ $=(0,-1)$ spectral component, even though the physical aspect ratio of HSX is $\sim 10$. In this sense, HSX looks like a straight stellarator in magnetic coordinates.

It is possible to make significant changes to the magnetic field spectrum of HSX using a set of auxiliary coils. Each of the 48 nonplanar coils has an adjacent planar coil, which can be used to add or subtract toroidal field from that produced by the main coils. These auxiliary coils can be operated in such a way that the magnetic field from sets of six auxiliary coils alternatively add to or subtract from the field of the main magnets. In this way, it is possible to introduce a large $(n, m)=(4,0)$ spectral component to the Hamada spectrum in addition to the $(4,1)$ helical modulation, as illustrated in Fig. 1(c). As will be shown later, this "mirror" configuration is predicted to have significantly larger neoclassical viscous damping than the QHS configuration, and in a general sense replicates the neoclassical transport of a conventional stellarator. The magnetic surfaces at the elliptical plane are illustrated in Fig. 1(b) for the QHS configuration and Fig. 1(d) for the mirror configuration, showing that the surface shape is similar for the two cases. The rotational transform, plasma volume, and magnetic well depth are not significantly different between these two configurations.

We have performed experiments and modeling in HSX to test the predicted reduction in flow damping with quasisymmetry. ${ }^{8}$ We have developed a fast-switching biased electrode system to spin-up the plasma. Using a system of Mach probes, we are able to measure the changes in plasma flow induced by the electrode system. These fast and localized flow measurements enable two time-scales in the flow evolution to be studied. Floating potential $\left(V_{\mathrm{f}}\right)$ time histories and radial profiles are measured as well, enabling both the time-scales for the $V_{\mathrm{f}}$ evolution and the steady state radial conductivity to be measured. We have performed these mea- surements in the base QHS configuration and in the mirror configuration, and have confirmed the reduction in flow damping with quasisymmetry.

We have used neoclassical theory, including the effects of neutrals, to model the steady state and time evolution of the plasma flow and electric field during electrode bias. The steady state solution of the momentum and continuity equations predicts the steady state radial conductivity. We model the spin-up with a model where the electric field formation initiates the spin-up. We model the decay using the formalism of Coronado and Talmadge, ${ }^{12}$ where the open-circuiting of the electrode current initiates the relaxation of the plasma flows and electric field. The modeled predictions about the radial conductivity, flow and electric field evolution timescales, and flow directions are all tested against measurements.

The time-scales for the electric field and plasma flow evolution when the bias is turned on are in good agreement with the neoclassical model. The steady state electrode current is significantly larger than the neoclassical prediction, indicating that the steady state radial conductivity is anomalously large. The measured slow time-scale for the decay of the flows is faster than the neoclassical prediction.

HSX is a medium size, four field-period stellarator experiment at the University of Wisconsin-Madison. HSX has a major radius of $1.2 \mathrm{~m}$ and a minor radius of $0.09-0.13 \mathrm{~m}$, depending on the machine configuration. The magnetic field on axis is $0.5 \mathrm{~T}$ in the experiments described in this paper. Plasma is produced and heated using approximately $50 \mathrm{~kW}$ of second harmonic electron cyclotron heating $(\mathrm{ECH})$ at $28 \mathrm{GHz}$. Hydrogen is the working gas in the experiments presented in this paper. For all mirror configuration data presented here, the auxiliary coils are energized with $10 \%$ of the amp-turns of the main modular coils. This mirror amplitude yields a configuration with neoclassical transport similar to the lower levels accessible in classical stellarators, though still much degraded compared to the QHS configuration.

The remainder of this paper is organized as follows. Section II will briefly describe the diagnostics and tools used in this research. Section III will present the measured evolution during and after bias of the electrode voltage and current, the floating potential, and the plasma flow. Section IV will describe the neoclassical modeling, with emphasis upon the techniques developed in this research. Section V will make comparisons between the neoclassical modeling and the measurements. Our conclusions are presented in Sec. VI.

\section{DIAGNOSTICS AND EXPERIMENTAL METHODS}

A biased electrode system ${ }^{13,14}$ is used to generate plasma rotation in HSX. A schematic of the experimental design is shown in Fig. 2. The electrode is inserted to $\sim 3 \mathrm{~cm}$ inside the last closed magnetic surface (LCMS) to $r / a \approx 0.65$ and biased positive with respect to the vacuum vessel. The current drawn from the plasma by the electrode flows down the shaft of the probe, through the power supply to the vacuum vessel, and then back through the plasma to the magnetic surface where the probe resides. This "return current" flow- 


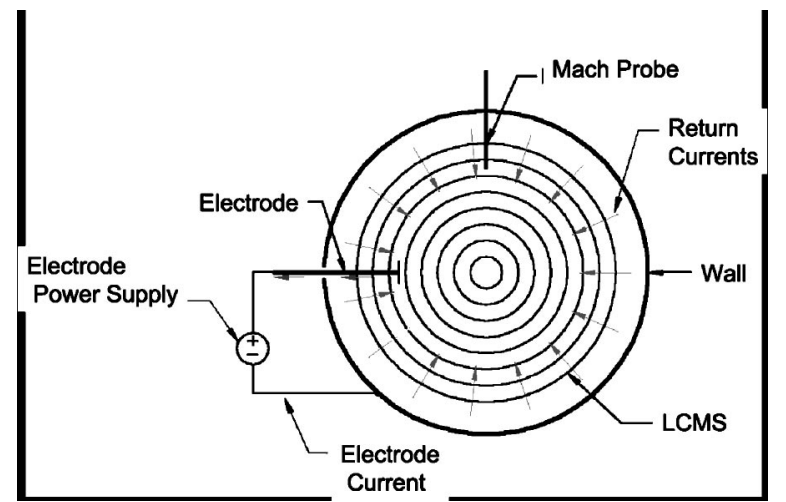

FIG. 2. The conceptual layout of the experiments presented in this paper. A current flowing through the plasma, induced by the biased electrode, causes a force that leads to plasma rotation.

ing through the plasma exerts a $\mathbf{J} \times \mathbf{B}$ torque to spin the plasma. At steady state, the torque exerted by this current must be equal to the damping forces. Details of the various components in the figure are provided below.

The electrode used in HSX is a molybdenum cylinder $2 \mathrm{~cm}$ in diameter, with $0.635 \mathrm{~cm}$ exposed beyond a boron nitride shroud. The power supply is composed of a $10 \mathrm{mF}$ capacitor bank capable of supplying $150 \mathrm{~A}$ at $500 \mathrm{~V}$ for short pulses, although the plasma seldom supplies more than $20 \mathrm{~A}$. The electrode voltage is switched on and the electrode current is turned off using solid state switches (Insulated Gate Bipolar Transistors, or IGBTs); typical voltage turn-on and current turn-off times are $1 \mu$ s. The voltage on the electrode is monitored at $100 \mathrm{kHz}$, while a Pearson current transformer monitors the electrode current with $\approx 1 \mathrm{MHz}$ bandwidth. The detailed design of the electrode and bias power supply has been presented elsewhere. ${ }^{15}$

To measure the changes in the plasma flow and floating potential when the electrode is energized, a set of Mach probe diagnostics have been developed. ${ }^{15}$ The probes, which are similar to the Gundestrup probes in the Tokamak de Varennes ${ }^{16}$ and Texas Experimental Tokamak, ${ }^{17}$ have six tips facing outward from the insulating body of the probe. The six tips are biased to ion saturation current $\left(I_{\text {sat }}\right)$ at $-180 \mathrm{~V}$ using battery packs. The signal is passed through isolation amplifiers with $100 \mathrm{kHz}$ of bandwidth before digitization. A seventh pin protrudes from the front of the probe (the "proud" pin), enabling the floating potential $V_{\mathrm{f}}$ to be monitored at the location of the probe with a bandwidth of $\approx 100 \mathrm{kHz}$. The probe is inserted approximately orthogonally to the magnetic surface, so that the flows measured are in the magnetic surface, i.e., we measure toroidal and poloidal flows, but not radial flows.

If the plasma were not flowing, then all six tips would draw the same ion saturation current; plasma flow causes the tips facing in the direction of the flow to collect more current than those facing away. This is illustrated in Fig. 3, where the raw data from the probe is illustrated for two different time slices. The data at $t=817 \mathrm{~ms}$ is for a time when the electrode bias is off, while the data for $t=819 \mathrm{~ms}$ is during the electrode bias. Note that the points are drawn down and to the

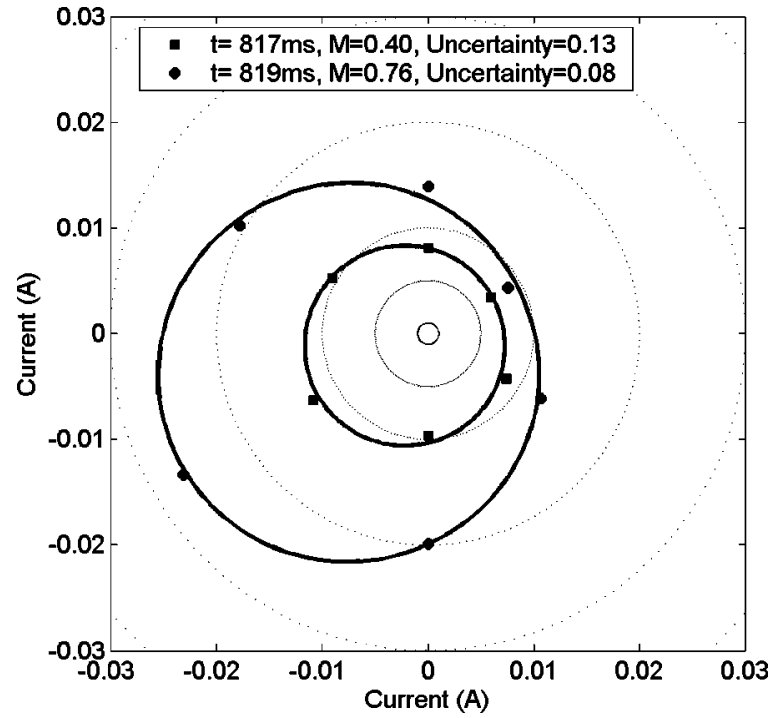

FIG. 3. Examples of the Mach probe data at two different time slices in a discharge. The data at $t=817 \mathrm{~ms}$ correspond to a time before the electrode bias is applied, and the increase in plasma flow during bias at $t=819 \mathrm{~ms}$ is evidenced by the set of $I_{\text {sat }}$ points being drawn down and to the left. The solid lines represent fits of the Hutchinson model to the raw data.

right during bias, indicating that the plasma flow comes from that direction.

A model is required to relate the asymmetry in collected $I_{\text {sat }}$ to the flow speed of the plasma. ${ }^{18}$ In our case, the data are analyzed using the unmagnetized model by Hutchinson. ${ }^{19}$ At each point in time, the six measurements are fit to a curve of the form

$$
\begin{aligned}
I_{\mathrm{sat}}\left(\theta ; A, M, \theta_{\mathrm{F}}\right)= & A \exp \left(\mathrm { M } \left\{\left[1-\cos \left(\theta-\theta_{\mathrm{F}}\right)\right] K_{\mathrm{u}}\right.\right. \\
& \left.\left.-\left[1+\cos \left(\theta-\theta_{\mathrm{F}}\right)\right] K_{\mathrm{d}}\right\} / 2\right) .
\end{aligned}
$$

with $K_{\mathrm{u}}=0.64$ and $K_{\mathrm{d}}=0.7$, and $A, M$, and $\theta_{\mathrm{F}}$ are the fit parameters, and $\theta$ is the angle of each of the six tips. The average $I_{\text {sat }}$ collected by the six tips is represented by the parameter $A, M$ is the Mach number of the flow [in this case, actual speed divided by $C_{\mathrm{s}}=\left(T_{\mathrm{e}} / m_{i}\right)^{1 / 2}$, with $T_{\mathrm{e}}$ the electron temperature and $m_{i}$ the ion mass], and $\theta_{\mathrm{F}}$ is the angle of the flow. This angle is rotated so that $\theta_{\mathrm{F}}=0$ is approximately the direction of the magnetic field. Examples of these fits are presented as the solid lines in Fig. 3. This fit is done at each time point, with the final values of $A, M$, and $\theta_{\mathrm{F}}$ stored in the HSX database as a function of time. Unless otherwise stated, flow velocities will be left in terms of Mach number and not converted to actual speed.

Two of these probes have been constructed for use on HSX. One is located on the low $|B|$ side of the machine and the other on the high $|B|$ side. Note that due to the helical nature of the $|B|$ contours, both of these locations are on the outboard side of the torus but separated by half of a field period. For all measurements illustrated in this paper, the radial location of the Mach probes is between the LCMS and the surface on which the electrode resides, as illustrated schematically in Fig. 2. This is the region over which the electrode voltage is dropped and through which the return current is flowing. 


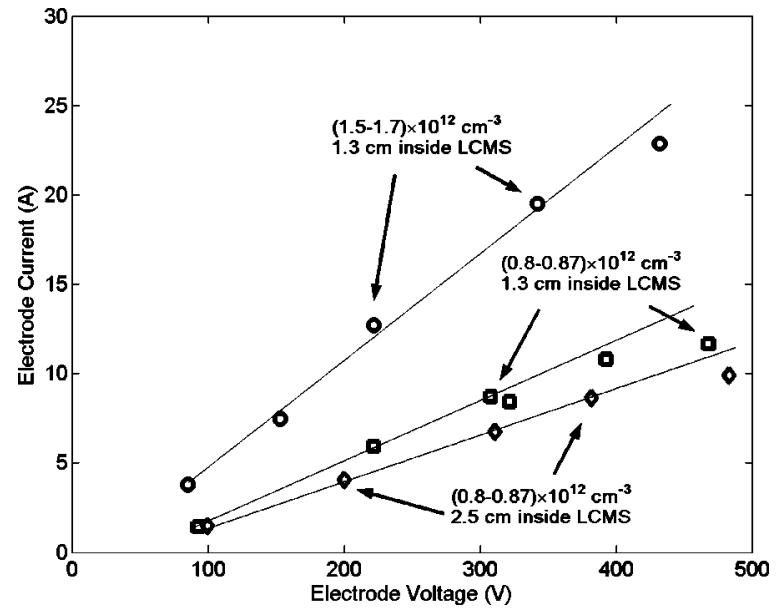

FIG. 4. The $I-V$ curve of the electrode for positive bias for three different combinations of electrode location and plasma density. Increasing the plasma density leads to more current at a fixed electrode location, while inserting the probe more deeply leads to less electrode current for a fixed line average plasma density.

Assessing the ion-neutral friction contribution to flow damping is an important goal of these studies. To monitor the neutral hydrogen density $\left(N_{\mathrm{n}}\right), 16$ absolutely calibrated $\mathrm{H}_{\alpha}$ detectors have been installed on HSX. ${ }^{20}$ Seven of these detectors have been installed around the torus in a toroidal array, while nine of them view the plasma at constant toroidal angle in a vertical array. The neutral gas code DEGAS ${ }^{21}$ is used to estimate the density of both atomic and molecular hydrogen in HSX, based on measured $\mathrm{H}_{\alpha}$ emission, the electron density profile, ${ }^{22}$ and the electron temperature profile. The details of the DEGAS calculation and comparison to the $\mathrm{H}_{\alpha}$ measurements will be presented elsewhere.

We estimate the ion temperature $T_{\mathrm{i}}$ using ion Doppler spectroscopy. An optical fiber coupled spectrometer is used to examine UV emission from intrinsic impurities on a chord passing through the magnetic axis. The instrument is a $1 \mathrm{~m}$ Czerny-Truner spectrometer with a 3600 grooves $/ \mathrm{mm}$ grating of dimensions $10 \times 10 \mathrm{~cm}^{2}$. The dispersed light is detected with a charge-coupled device (CCD) detector; a single exposure of the CCD can be obtained during a discharge. The $\mathrm{O}^{4+}$ line at $278.101 \mathrm{~nm}$ and the $\mathrm{O}^{1+}$ line at $278.993 \mathrm{~nm}$ are routinely used for ion temperature measurements. For densities of $1 \times 10^{12} \mathrm{~cm}^{-3}$ and $50 \mathrm{~kW}$ launched power, we measure $T_{\mathrm{i}}=20 \mathrm{eV}$ for both spectral lines, in both QHS and mirror configurations. This $T_{\mathrm{i}}$ is sufficient to place the ions in the plateau regime. Further, the similar temperatures for $\mathrm{O}^{4+}$ and $\mathrm{O}^{1+}$ give some indication that the ion temperature profile is fairly flat, in agreement with preliminary power balance calculations. Note that the proton temperature and the impurity ion temperature are calculated to be quite similar, due to the tight proton-impurity collisional coupling.

\section{THE PHENOMENOLOGY OF BIASED DISCHARGES IN HSX}

The $I-V$ curve of the electrode for positive bias is shown in Fig. 4 for three different cases in the QHS configuration. At fixed electrode location (1.3 cm inside the LCMS), the

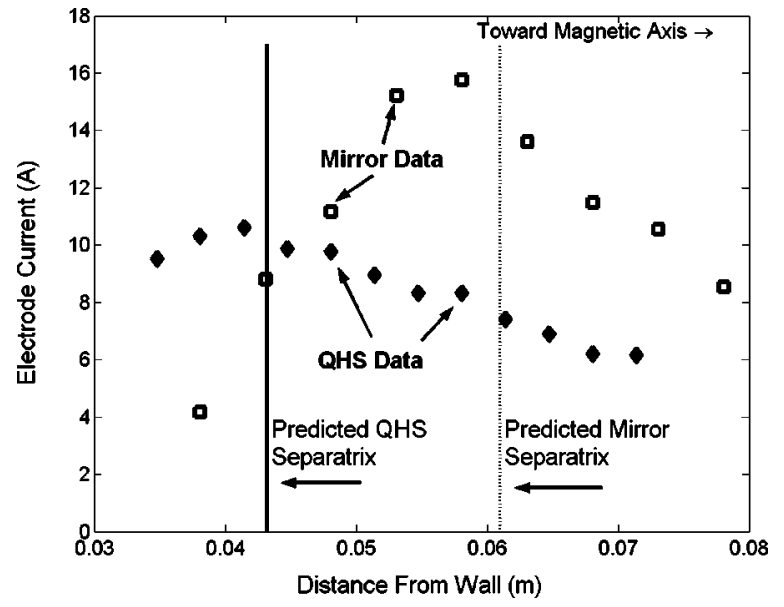

FIG. 5. Variation of electrode current with electrode location for the QHS and mirror configurations. The line average plasma density $\left(1 \times 10^{12} \mathrm{~cm}^{-3}\right)$ and electrode voltage $(340 \mathrm{~V})$ are held fixed in these scans. Note the peak in electrode current at the location of the separatrix.

$I-V$ characteristic was measured at line average densities of $0.8 \times 10^{12} \mathrm{~cm}^{-3}$ and $1.6 \times 10^{12} \mathrm{~cm}^{-3}$. These curves show the expected increase in electrode current with plasma density for all bias voltages. The figure also shows a $I-V$ curve for a line average density of $0.8 \times 10^{12} \mathrm{~cm}^{-3}$ with the electrode positioned $2.5 \mathrm{~cm}$ inside the separatrix.

The data in Fig. 4 shows only positive bias cases. We have found that for negative bias, the voltage drop at the electrode sheath is a large fraction of the total voltage. The amount of voltage dropped across the plasma is correspondingly small, and the electrode current is reduced. For these reasons, the data presented in this paper will be for positive bias only. Note that the $I-V$ curves are approximately straight lines, except for possibly a small roll over at the highest bias voltages which are accessible with the system. We have not observed any region of negative resistance (where increasing the electrode voltage decreases the electrode current), an observation that will provide justification for the use of linear neoclassical viscosities in modeling described in Sec. IV. It is entirely possible that biasing to larger voltages or operating with different plasma parameters (electron and ion temperatures, plasma density,...) could lead to a bifurcation, ${ }^{13,14}$ but this has not been observed to date.

We observe that the electrode current peaks when the electrode is located at the LCMS, and decreases as the probe is inserted more deeply. This is shown in Fig. 5, where the electrode location was scanned on a shot to shot basis from outside to inside the LCMS, with fixed electrode voltage and line average density. The scan is shown for both the QHS and the mirror configurations. In both cases a local maxima in the electrode current occurs at the separatrix. As the probe is moved away from the separatrix and toward the magnetic axis, the electrode current decreases in spite of the fact that the electrode is moving into regions of higher plasma density.

The gas puff was adjusted before each discharge so that the central chord of the multichord interferometer was constant during these scans of the electrode location. Considering the QHS case particularly, subsequent data analysis 
showed that there were not substantial changes in the plasma density along any of the interferometer chords as the probe was inserted. Hence, we infer that the electrode is not acting as a limiter. The stored energy decreases by about $20 \%$ as the electrode is driven from the edge to $r / a=0.6$. The $H_{\alpha}$ emission at the location of the gas puff valve and at the electrode was constant to within $25 \%$ throughout the entire scan. Further, the chord integrated $\mathrm{O}^{+4}(278.1 \mathrm{~nm})$ temperature was constant at $\approx 20 \mathrm{eV}$ throughout the scan. Hence, we infer that the decrease in electrode current as the electrode is moved in is not due to a degradation of the plasma parameters or change in profiles.

A more plausible explanation is that the electrode current is limited by cross-field transport. The current drawn by the electrode must be balanced by a return current in steady state. As will be described in Sec. IV, this return current is related to the electric field in steady state through the radial conductivity,

$$
\left\langle\mathbf{J}_{\text {plasma }} \cdot \boldsymbol{\nabla} \psi\right\rangle=\sigma_{\perp}\left(\frac{d \Phi}{d \psi}\langle\nabla \psi \cdot \nabla \psi\rangle-\frac{\left\langle\boldsymbol{\nabla} p_{\mathrm{i}} \cdot \boldsymbol{\nabla} \psi\right\rangle}{e N_{\mathrm{i}}}\right) .
$$

Here, $\mathbf{J}_{\text {plasma }}$ is the current density flowing through the plasma, $\psi$ is the toroidal flux, $p_{\mathrm{i}}$ is the ion pressure, $e$ is the elementary charge, $\Phi$ is the potential of the plasma, $N_{\mathrm{i}}$ is the plasma density, $\sigma_{\perp}$ is the radial conductivity, and $\langle\cdots\rangle$ represents an average over the magnetic surface. ${ }^{23}$ Neglecting the ion pressure gradient compared to the electric field under bias conditions, the electrode current can be related to the potential gradient as

$$
\frac{d \Phi}{d \psi}=\frac{I_{\text {electrode }}}{\sigma_{\perp} A_{\text {surf }}} \frac{\langle|\nabla \psi|\rangle}{\langle\nabla \psi \cdot \nabla \psi\rangle},
$$

where $A_{\text {surf }}$ is the area of the magnetic surface. Integrating this expression from the edge (where the potential is $\Phi_{\mathrm{a}}$ ) to the surface with the electrode allows an effective impedance to be defined as

$$
R_{\text {eff }}=\int_{\psi_{\mathrm{a}}}^{\psi_{\text {electrode }}} \frac{1}{\sigma_{\perp} A_{\text {surf }}} \frac{\langle|\nabla \psi|\rangle}{\langle\nabla \psi \nabla \psi\rangle} d \psi
$$

All of the terms in the integrand in Eq. (5) are positive, so that increasing the depth of electrode insertion will increase resistance seen by the electrode. At fixed electrode voltage, this will cause the electrode current to decrease. Detailed measurements and modeling of the radial conductivity are presented in Secs. IV and V.

Representative profiles of the floating potential $V_{\mathrm{f}}$ are shown in Fig. 6, where the measurements were made with the proud pin of the Mach probe on a shot to shot basis. Curves are shown for bias voltage of $0 \mathrm{~V}$ (electrically floating electrode, before bias), $350 \mathrm{~V}$, and $490 \mathrm{~V}$. For each voltage, measurements from the high and low field side of the torus are displayed, where the radial location of the measurement has been mapped to toroidal flux for display in the figure. At fixed bias voltage, the profile measurements made on the high and low $|B|$ side of the torus agree well. The approximate location of the biased electrode is shown in the

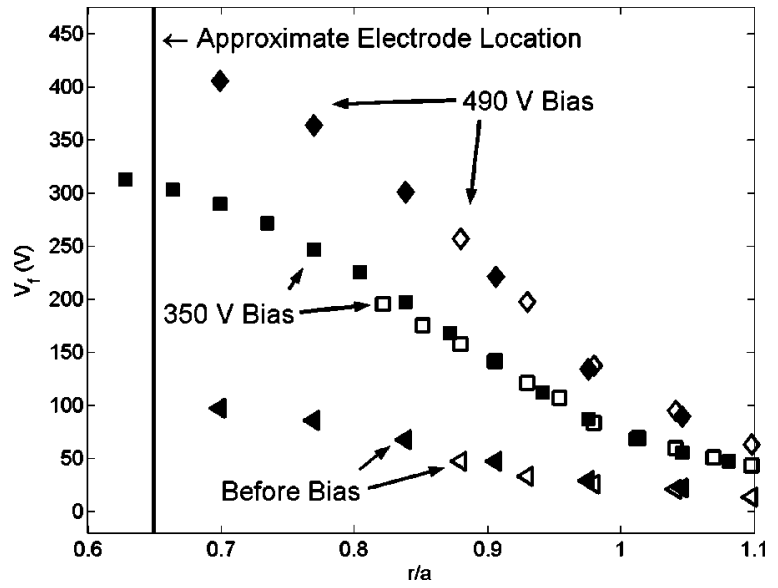

FIG. 6. Profiles of the floating potential in the QHS configuration for bias voltages of $0 \mathrm{~V}, 350 \mathrm{~V}$, and $490 \mathrm{~V}$. Closed symbols correspond to measurements with the low field side Mach probe, while open symbols are measurements from the high field side.

figure as well, and the measurements indicate that the bias voltage is smoothly dropped from the LCMS to the location of the electrode.

We next turn to the time evolution of the electrode voltage and current and the floating potential. The evolution of these quantities during and after the electrode pulse is shown in Fig. 7. The electrode is located at $r / a=0.65$ for the QHS discharge displayed here, and the line average density is 1 $\times 10^{12} \mathrm{~cm}^{-3}$. In making this and most subsequent plots, between 12 and 24 similar bias electrode pulses are averaged to reduce the noise in the signals.

An important feature to note is the asymmetry between the turn-on and turn-off of the electrode. The electrode voltage in Fig. 7(a) is applied in $\sim 1 \mu$ s. The electrode current in Fig. 7(b) responds to the application of this voltage by drawing a very large current spike before settling to its steady state value. The electrode current is terminated in $\sim 1 \mu$ s at the end of the electrode pulse. Once the electrode current is broken, the electrode acts like a large floating potential monitor. The floating potential measured by the electrode decays on a time-scale of typically $\sim 30 \mu \mathrm{s}$.

The floating potential evolution at the proud pin of the Mach probe is shown in Fig. 7(c). The floating potential is

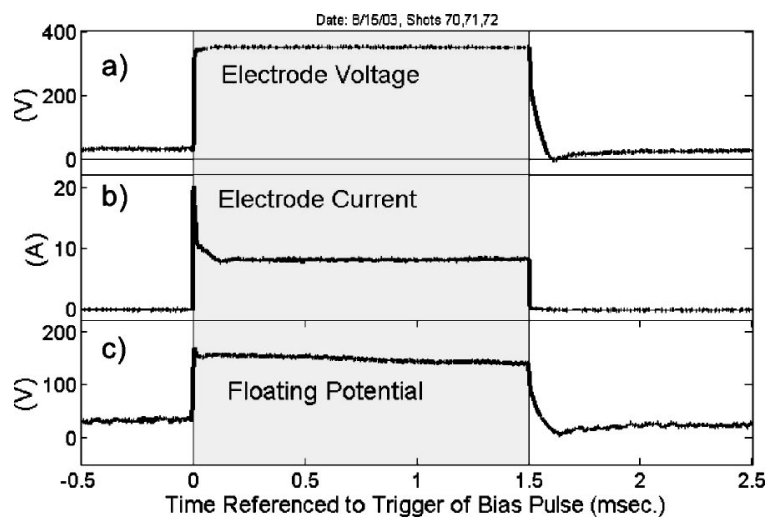

FIG. 7. Time evolution of (a) the electrode voltage, (b) the electrode current, and (c) the floating potential during and after electrode bias. 


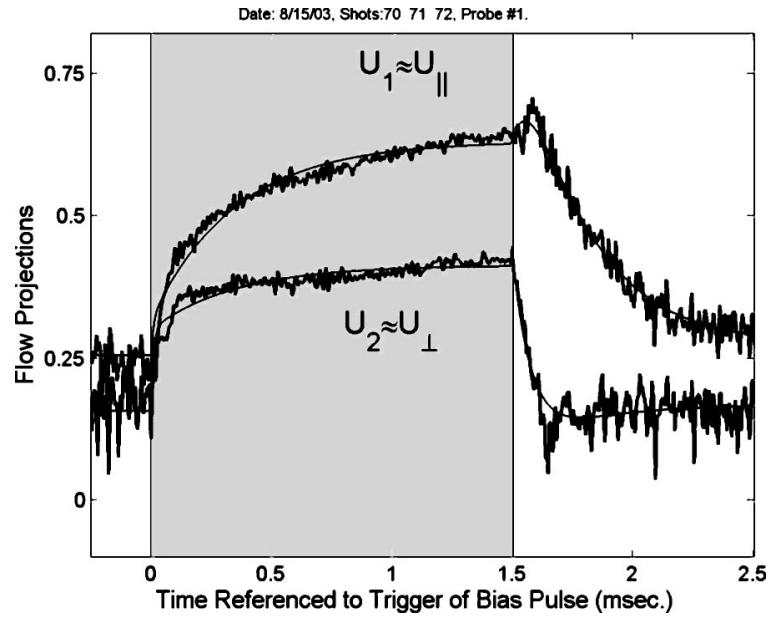

FIG. 8. The evolution of the approximately parallel $\left(U_{1}\right)$ and perpendicular $\left(U_{2}\right)$ flows during and after the bias pulse. Thin black lines correspond to the fits of Eq. (8) to the spin-up and relaxation phases.

measured at $r / a \approx 0.87$, a location inside the LCMS but outside the surface where the bias electrode resides. The floating potential evolution appears as essentially a scaled version of the electrode voltage. There is a very fast rise when the electrode voltage is applied, a duration at a near stationary level, and a decay with a time-scale of a few tens of microseconds. These results show similarity to limiter biasing experiments in TJ-II, ${ }^{24}$ where a fast time-scale $(\sim 50 \mu \mathrm{s})$ was observed in the decay of the floating potential after the fast termination of bias. This time-scale is similar to that observed in HSX. A slower time-scale in the floating potential evolution $(\sim 10 \mathrm{~ms})$, related to the slower evolution of density and temperature, was also observed in the TJ-II experiments. This time-scale is not observed for the HSX results presented here, probably because the bias pulses are kept sufficiently short that no significant evolution of the plasma density is allowed to occur.

The typical plasma flow evolution during and after the bias pulse is illustrated in Fig. 8. Recall that the Mach probe produces data in the form of the flow speed and angle as a function of time. The data in the figure have been cast in term of the orthogonal projections of the flow by calculating

$$
\begin{aligned}
& U_{1, \exp }(t)=M(t) \cos \left[\theta_{\mathrm{F}}(\mathrm{t})\right], \\
& U_{2, \exp }(t)=M(t) \sin \left[\theta_{\mathrm{F}}(\mathrm{t})\right] .
\end{aligned}
$$

Since the flow angle was rotated so that $\theta_{\mathrm{F}}=0$ is approximately parallel to the magnetic field, $U_{1}$ is the approximately parallel flow and $U_{2}$ is the flow which is in the magnetic surface but perpendicular to the magnetic field. Note that the parallel flow $\left(U_{1}\right)$ has a much longer evolution than the perpendicular flow $\left(U_{2}\right)$, for both the spin-up and decay phases.

We analyze the flow evolution data using a two-timescale ansatz, based on the two-time-scale observation presented above and neoclassical theory to be presented in Sec. IV. If the biased electrode is turned on at $t=0$, then the flow evolution for $t>0$ will be of the form

$$
\mathbf{U}(t)=C_{f}\left[1-\exp \left(-r_{f} t\right)\right] \mathbf{f}+C_{s}\left[1-\exp \left(-r_{s} t\right)\right] \mathbf{s}+\mathbf{U}_{s s},
$$

where $\mathbf{f}$ and $\mathbf{s}$ are unit vectors in the fast and slow rising directions, $C_{\mathrm{f}}$ and $C_{\mathrm{s}}$ are the amount of flow in those directions, $r_{\mathrm{f}}$ and $r_{\mathrm{s}}$ are the fast and slow rise rates, and $\mathbf{U}_{s s}$ is the flow before the bias is turned on. By projecting this equation parallel and perpendicular to the field, it can be compared to the measured flow projections and used as a fitting function. Hence, the projections $U_{1 \text {,exp }}$ and $U_{2 \text {,exp }}$ are fit to functions of the form

$$
\begin{aligned}
U_{1, f i t}(t)= & C_{f}\left[1-\exp \left(-r_{f} t\right)\right] \cos \left(\alpha_{f}\right) \\
& +C_{s}\left[1-\exp \left(-r_{s} t\right)\right] \cos \left(\alpha_{s}\right)+U_{1 S S}, \\
U_{2, f i t}(t)= & C_{f}\left[1-\exp \left(-r_{f} t\right)\right] \sin \left(\alpha_{f}\right) \\
& +C_{s}\left[1-\exp \left(-r_{s} t\right)\right] \sin \left(\alpha_{s}\right)+U_{2 S S},
\end{aligned}
$$

where $\alpha_{f}$ and $\alpha_{s}$ are the angles of fast and slow flow unit vectors with respect to the magnetic field. The $C$ 's, $\tau$ 's, and $\alpha$ 's are free parameters determined by a nonlinear fitting routine, as are $U_{1 \mathrm{SS}}$ and $U_{2 \mathrm{Ss}}$. A similar two-direction/two-timescale fit is applied to the decay of the flow by making the substitution in Eq. (8): $[1-\exp (-r t)] \rightarrow \exp \left[-r\left(t-t_{\mathrm{o}}\right)\right]$, where $t_{\mathrm{o}}$ is the time when the electrode current is broken.

An example of these fits is superimposed on the data in Fig. 8. The projections illustrate that there are two timescales involved in the flow evolution, with the fast time-scale appearing most strongly in $U_{2}$ and the slow time-scale appearing more strongly in $U_{1}$. The fits of the form in Eq. (8) are shown as the thin lines, and yield a fast rise time of $\approx 10 \mu$ s and a slow rise time of $\approx 450 \mu$ s. Caution should be observed in interpreting the fast time of the flow rise, as the bandwidth of the measuring electronics is only $100 \mathrm{kHz}$.

The flow decay also exhibits a two-time-scale behavior. The fast decay time is $\approx 50 \mu \mathrm{s}$, and is mainly visible in $U_{2}$, the perpendicular component of the flow. The slow decay time is typically a few hundred microseconds, and is mainly present in the parallel flow. Note that the fast rise time is generally faster than the fast decay time.

These time-scales are to be compared with the rise and decay times of the local floating potential presented earlier in this section. The floating potential rise occurs in $\approx 5 \mu \mathrm{s}$, a time-scale commensurate with the fast flow rise. At the end of the electrode pulse, the floating potential decays in $\approx 40 \mu \mathrm{s}$. This is a similar time-scale to the fast flow decay when the electrode current is shut off. Hence, the floating potential and the perpendicular parts of the flows decay on a time-scale of $\sim 30-50 \mu$ s when the electrode current is broken, while the parallel flows tend to decay on a time-scale of a few hundred microseconds. There is no indication of the slower time-scale in either the potential rise or fall.

These measurements have been made in otherwise similar QHS and mirror discharges. The flow speed evolution (as a Mach number) is shown for representative QHS and mirror discharges in Fig. 9. These two discharges have the same line average density and $\mathrm{ECH}$ power, and the electrode and probes are at similar locations. The electrode voltage was $\sim 340 \mathrm{~V}$ in both cases. The two waveforms have a similar 


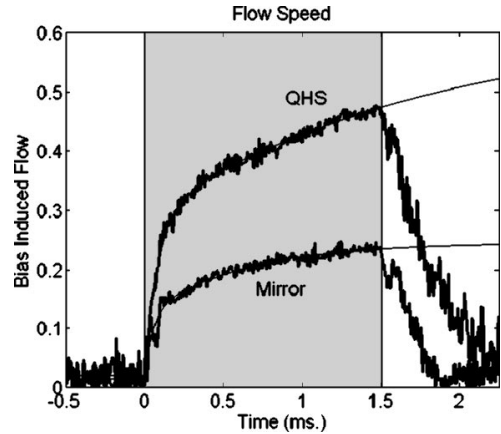

FIG. 9. Time evolution of the Mach number in similar QHS and mirror configuration discharges. The flow in the QHS case takes longer to spin-up, reaches a higher final speed, and decays more slowly.

initial rise, indicating that the fast component of the flow rise is similar for the two configurations. After this fast rise, the QHS wave form continues to climb, even as the mirror wave form saturates at a lower level. The flow speed in the QHS configuration takes significantly longer to reach a steady state value, and the steady state value of the flow is larger for the QHS configuration. The current drawn by the electrode in steady state was $8 \mathrm{~A}$ in the QHS configuration, while the value for the mirror configuration was $10 \mathrm{~A}$. Hence, the QHS configuration has more total flow per unit of $\mathbf{J} \times \mathbf{B}$ torque. When the electrode current is terminated, the QHS case takes longer for the flow to decay than the mirror. All of these features indicate a reduction in damping with quasisymmetry. More detailed comparisons of damping measurements between these two configurations will be given in Sec. V.

In the past, many biasing experiments have focused on the impact of biasing on turbulence and the transport of particles and heat. ${ }^{13,14,25-27}$ We have observed a decrease in floating potential and $I_{\text {sat }}$ fluctuation amplitude (up to a frequency of $100 \mathrm{kHz}$ ) during bias, ${ }^{8}$ but have yet to make the detailed turbulence and profile measurements necessary to draw firm conclusions about the impact of bias on transport. On the other hand, comparisons of QHS and mirror plasmas without bias have already demonstrated the reduction in orbit deviations from a flux surface ${ }^{28}$ and the suppression of direct losses $^{29}$ in the QHS configuration, compared to the mirror case.

\section{MODELING THE PLASMA FLOW AND ELECTRIC FIELD EVOLUTION}

HSX is not perfectly symmetric; the small symmetry breaking terms visible in Fig. 1(a) will contribute to neoclassical damping of the flows. Furthermore, the low electron density of the ECH-heated plasmas allows significant neutral penetration. Hence, it is reasonable to hypothesize that neoclassical effects, including the effects of neutral friction, may be responsible for the measured flow damping in HSX. We have taken the approach that a careful calculation of neoclassical viscous damping is an important first step in understanding flow damping in HSX.

This neoclassical formulation involves solving the continuity and momentum balance equations on a magnetic surface as a function of time. The description provided in this section provides minimal details where we have simply taken results from the original work by Coronado and Talmadge ${ }^{12}$ topics that have been developed in the course of the current research are presented in more detail. Section IV A details the basic equations and steady state solution for the radial conductivity and flow direction. Section IV B describes the model for the decay of the plasma flows and electric field, while Sec. IV C provides the details of the spin-up model. A comparison of the spin-up and relaxation models is provided in Sec. IV D.

These calculations are done in Hamada coordinates, ${ }^{23}$ where the Jacobian $\sqrt{ } g$ is a magnetic surface constant and both the magnetic field and the equilibrium MHD currents are straight lines. Note that in the original paper, ${ }^{12}$ the magnetic surface angles varied between 0 and 1 , while we allow them to vary from 0 to $2 \pi$. The original paper also used the volume $V$ as a flux label, while we leave the magnetic surface label as an arbitrary variable $\rho$. These assumptions lead to a Jacobian equal to 1 in the earlier work, while it is equal to $\left(1 / 4 \pi^{2}\right) \partial V / \partial \rho$ in the present case.

\section{A. Basic equations and the steady state solutions}

The lowest order continuity equation yields $\boldsymbol{\nabla} \cdot \mathbf{U}=0,{ }^{30}$ while the lowest order momentum balance equation yields

$$
\mathbf{U}_{\perp}=-c\left(\frac{\partial \Phi}{\partial \rho}+\frac{1}{e N_{\mathrm{i}}} \frac{\partial \mathrm{p}_{\mathrm{i}}}{\partial \rho}\right) \frac{B \times \nabla \rho}{B^{2}} .
$$

These two results can be used to write the contravariant poloidal and toroidal flows in Hamada coordinates as ${ }^{31}$

$$
\begin{aligned}
U^{\alpha} & =\frac{c}{B^{\zeta} \sqrt{g}}\left(\frac{\partial \Phi}{\partial \rho}+\frac{1}{e N_{\mathrm{i}}} \frac{\partial p_{\mathrm{i}}}{\partial \rho}\right)+\lambda B^{\alpha}, \\
U^{\zeta} & =\lambda B^{\zeta} .
\end{aligned}
$$

In these expressions, $N_{\mathrm{i}}$ is the ion density, $c$ is the speed of light, and $B^{\alpha}$ and $B^{\zeta}$ are the Hamada contravariant poloidal and toroidal fields, respectively. The force free parallel flow $\lambda$ is a magnetic surface constant analogous to the "bootstrap" current.

The first-order parallel and poloidal momentum balance equations are given by

$$
\begin{aligned}
m_{\mathrm{i}} N_{\mathrm{i}} \frac{\partial}{\partial t}\langle\mathbf{B} \cdot \mathbf{U}\rangle= & -\langle\mathbf{B} \cdot \boldsymbol{\nabla} \cdot \Pi\rangle-m_{\mathrm{i}} N_{\mathrm{i}} v_{\mathrm{in}}\langle\mathbf{B} \cdot \mathbf{U}\rangle \\
m_{i} N_{i} \frac{\partial}{\partial t}\left\langle\mathbf{B}_{P} \cdot \mathbf{U}\right\rangle= & -\frac{\sqrt{g} \boldsymbol{B}^{\zeta} \boldsymbol{B}^{\alpha}}{c}\left\langle\mathbf{J}_{\text {plasma }} \cdot \boldsymbol{\nabla} \rho\right\rangle \\
& -\left\langle\mathbf{B}_{P} \cdot \boldsymbol{\nabla} \cdot \Pi\right\rangle-m_{i} N_{i} v_{i n}\left\langle\mathbf{B}_{P} \cdot \mathbf{U}\right\rangle .
\end{aligned}
$$

Here, $\mathbf{J}_{\text {plasma }}$ is a current flowing through the plasma, causing a force that drives the plasma flow.

In calculating the ion-neutral collision frequency in the neoclassical modeling, we use the momentum transfer crosssection data from the quantum-mechanical calculation by Krstic and Schultz. ${ }^{32}$ Their calculation includes both charge exchange and classical elastic scattering, and allows accurate calculation of the momentum transfer rates through the $\Omega$-integral ${ }^{33}$ formulation. 
For a quick estimate of the ion-neutral collision frequency, $v_{\text {in }} \approx N_{\mathrm{n}} 10^{-8} T_{\mathrm{i}}^{318}$ from Cornelis et al., ${ }^{34}$ provides a reasonable approximation for the momentum loss rate due to ion-neutral friction. For simplicities sake, $v_{\text {in }}$ has been factored out of the flux-surface averages in Eqs. (11) and (12). This step is not strictly accurate for HSX, where the localized gas-puff source leads to a large toroidal asymmetry in the neutral density. Methods to include the proper distribution of neutrals on a magnetic surface have been developed, ${ }^{8}$ but the modification does not lead to a substantial change in the results of the modeling. Hence, the flux-surface average neutral density will be used in Eqs. (11) and (12) throughout the modeling presented in this paper.

To relate the current flowing in the plasma to the external current, we use the radial component of Ampere's law

$$
\frac{\partial}{\partial t} \frac{\partial \Phi}{\partial \rho}\langle\boldsymbol{\nabla} \rho \cdot \nabla \rho\rangle=4 \pi\left\langle\mathbf{J}_{\text {plasma }} \cdot \nabla \rho\right\rangle+\left\langle\mathbf{J}_{\text {ext }} \cdot \nabla \rho\right\rangle .
$$

The external current represents the current drawn by the electrode in the case under consideration in this paper, but could in general represent other nonambipolar particle transport mechanisms.

The neoclassical viscosities in Eqs. (11) and (12) are analytically calculated for the plateau regime ${ }^{35,36}$ as

$$
\begin{aligned}
& \langle\mathbf{B} \cdot \boldsymbol{\nabla} \cdot \Pi\rangle=\mu_{\alpha} U^{\alpha}+\mu_{\zeta} U^{\varsigma}, \\
& \left\langle\mathbf{B}_{P} \cdot \boldsymbol{\nabla} \cdot \Pi\right\rangle=\mu_{\alpha}^{(P)} U^{\alpha}+\mu_{\zeta}^{(P)} U^{\varsigma},
\end{aligned}
$$

where $\quad \mu_{\alpha}=\kappa\left(B^{\alpha} \alpha_{\mathrm{P}}+B^{\zeta} \alpha_{\mathrm{C}}\right), \quad \mu_{\zeta}=\kappa\left(B^{\alpha} \alpha_{\mathrm{C}}+B^{\zeta} \alpha_{\mathrm{T}}\right), \quad \mu_{\alpha}^{(\mathrm{P})}$ $=\kappa B^{\alpha} \alpha_{\mathrm{P}}$, and $\mu_{\zeta}^{(\mathrm{P})}=\kappa B^{\alpha} \alpha_{\mathrm{C}}$. These expressions in turn use $\kappa$ $=\pi^{1 / 2} P B_{\mathrm{o}} / V_{\mathrm{ta}} B^{\zeta}, \quad \alpha_{\mathrm{T}}=\sum n^{2} b_{\mathrm{n}, \mathrm{m}}^{2} /|n-m t|, \quad \alpha_{\mathrm{P}}=\sum m^{2} b_{\mathrm{n}, \mathrm{m}}^{2} /|n-m t|$, and $\alpha_{\mathrm{C}}=-\sum n m b_{\mathrm{n}, \mathrm{m}}^{2} /|n-m t|$, where $P$ is the pressure, $v_{\mathrm{t}}$ is the thermal velocity, $t$ is the rotational transform, and the sums are over all spectral components except the $(n, m)=(0,0)$ component. Terms in the viscosities proportional to the heat flux are neglected in this formulation. Strictly speaking, these viscosities are only valid on time-scales longer than an ion-ion collision time $\left(\tau_{i i}\right)^{37}$ Thus, our neoclassical calculations are only valid for damping rates less than $1 / \tau_{i i}$ $<10 \mathrm{kHz}$ based on HSX parameters.

The steady state limits of Eqs. (11)-(13) allow the relationship between the electric field and radial current to be written as

$$
\left\langle\mathbf{J}_{\text {plasma }} \cdot \boldsymbol{\nabla} \rho\right\rangle=\sigma_{\perp}\left(\left\langle\mathbf{E}_{r} \cdot \boldsymbol{\nabla} \rho\right\rangle-\frac{\left\langle\boldsymbol{\nabla} p_{\mathrm{i}} \cdot \boldsymbol{\nabla} \rho\right\rangle}{e N_{\mathrm{i}}}\right),
$$

where the radial conductivity is calculated as

$$
\begin{aligned}
\sigma_{\perp}= & \frac{c^{2} m_{\mathrm{i}} N_{\mathrm{i}}\left\langle B_{\mathrm{P}}^{2}\right\rangle}{\langle\nabla \rho \cdot \nabla \rho\rangle\left(\sqrt{g} B^{\alpha} B^{\zeta}\right)^{2}\left(t v_{\alpha}+v_{\zeta}+v_{\text {in }} \frac{\left\langle B^{2}\right\rangle}{\left\langle B^{2}\right\rangle}\right)} \\
& \times\left[\left(v_{\alpha}^{(\mathrm{p})}+v_{\text {in }} \frac{\left\langle\mathbf{B}_{\mathrm{P}} \cdot \mathbf{B}_{\mathrm{P}}\right\rangle}{\left\langle B_{\mathrm{P}}^{2}\right\rangle}\right)\left(v_{\zeta}+v_{\text {in }} \frac{\left\langle\mathbf{B} \cdot \mathbf{B}_{\mathrm{T}}\right\rangle}{\left\langle B^{2}\right\rangle}\right)\right. \\
& \left.-\left(v_{\zeta}^{(\mathrm{P})}+t v_{\text {in }} \frac{\left\langle\mathbf{B}_{\mathrm{P}} \cdot \mathbf{B}_{\mathrm{T}}\right\rangle}{\left\langle\mathbf{B}_{\mathrm{P}}^{2}\right\rangle}\right)\left(v_{\alpha}+v_{\text {in }} \frac{\left\langle\mathbf{B} \cdot \mathbf{B}_{\mathrm{P}}\right\rangle}{t\left\langle\mathbf{B}^{2}\right\rangle}\right)\right] .
\end{aligned}
$$

The viscous frequencies in this equation are defined as $v_{\alpha}$ $=\mu_{\alpha} B^{\zeta} / m_{\mathrm{i}} N_{\mathrm{i}}\langle\mathbf{B} \cdot \mathbf{B}\rangle, \quad \boldsymbol{v}_{\zeta}=\mu_{\zeta} B^{\zeta} / m_{\mathrm{i}} N_{\mathrm{i}}\langle\mathbf{B} \cdot \mathbf{B}\rangle, \quad \boldsymbol{v}_{\alpha}^{(\mathrm{P})}=\mu_{\alpha}^{(\mathrm{P})} B^{\alpha} /$ $m_{\mathrm{i}} N_{\mathrm{i}}\left\langle\mathbf{B}_{\mathrm{P}} \cdot \mathbf{B}_{\mathrm{P}}\right\rangle$, and $v_{\zeta}^{(\mathrm{P})}=\mu_{\zeta}^{(\mathrm{P})} B^{\alpha} / m_{\mathrm{i}} N_{\mathrm{i}}\left\langle\mathbf{B}_{\mathrm{P}} \cdot \mathbf{B}_{\mathrm{P}}\right\rangle$. Comparisons between this neoclassical radial conductivity and measurements will be presented in Sec. V.

The neoclassical modeling also predicts the steady state plasma flow direction in Hamada coordinates. The prediction for the steady state flow is of the form $\mathbf{U}_{\mathrm{ss}}=U_{\mathrm{ss}}^{\zeta} \mathbf{e}_{\zeta}+U_{\mathrm{ss}}^{\alpha} \mathbf{e}_{\alpha}$, with

$$
\begin{aligned}
& U_{\mathrm{ss}}^{\zeta}=-K B^{\zeta}\left(t v_{\alpha}+v_{\mathrm{in}} \frac{\left\langle\mathbf{B} \cdot \mathbf{B}_{\mathrm{P}}\right\rangle}{\left\langle B^{2}\right\rangle}\right) . \\
& U_{\mathrm{ss}}^{\alpha}=K B^{\alpha}\left(v_{\zeta}+v_{\mathrm{in}} \frac{\left\langle\mathbf{B} \cdot \mathbf{B}_{\mathrm{T}}\right\rangle}{\left\langle B^{2}\right\rangle}\right) .
\end{aligned}
$$

In these expressions, the constant $K$ is related to the radial current as

$$
K=\frac{c\langle\mathbf{J} \cdot \nabla \rho\rangle}{\left(\sqrt{g} B^{\alpha} B^{\zeta}\right)\langle\nabla \rho \cdot \nabla \rho\rangle \sigma_{\perp}\left(t v_{\alpha}+v_{\zeta}+v_{\text {in }}\right)} .
$$

This prediction for the steady state flow direction will be compared to measurements in Sec. V.

The calculation of the viscosity coefficients requires calculation of the Fourier decomposition of $|B|$ in Hamada coordinates. This was done for HSX using a modification of the technique originally developed for the calculation of the Boozer spectrum. ${ }^{38}$ A sufficient number of spectral components are used in the calculation, such that adding additional terms does not change the values of the viscosity coefficients $\alpha_{T}, \alpha_{P}$, and $\alpha_{C}$.

The calculation of the neoclassical radial conductivity also requires terms such as $\left\langle\mathbf{B}_{\mathrm{P}} \cdot \mathbf{B}_{\mathrm{P}}\right\rangle,\left\langle\mathbf{B}_{\mathrm{T}} \cdot \mathbf{B}_{\mathrm{P}}\right\rangle$, and $\langle\mathbf{B} \cdot \mathbf{B}\rangle$. Furthermore, the flow direction predictions are written in terms of the contravariant toroidal and poloidal flows. To calculate the flux surface averages noted above and to compare these neoclassical flow direction predictions to labframe measurements, it is necessary to know the Hamada coordinate basis vectors. In previous modeling, the basis vectors for a large aspect ratio circular tokamak ${ }^{39}$ were used to approximate the stellarator basis vectors. Given that HSX has essentially no toroidal curvature, the use of the tokamak basis vector approximation is not justified. We have derived a method to calculate the Hamada basis vectors for an arbitrary torus ${ }^{8}$ and applied it to the QHS and mirror configuration of HSX. The calculation technique will be discussed elsewhere, but the results will be used in the neoclassical calculations and theory/experiment comparisons discussed below.

\section{B. The flow relaxation model}

The flow relaxation formulation presented in this section is based on the work of Coronado and Talmadge. ${ }^{12}$ To examine the time evolution of the flows and electric fields when the bias current is terminated, we first rewrite the fluid equations in terms of the force free flow $(\lambda)$ and the electric field $(d \Phi / \partial \rho)$. The poloidal momentum balance equation becomes 


$$
a_{1} \frac{\partial}{\partial t} \frac{\partial \Phi}{\partial \rho}+a_{2} \frac{\partial \lambda_{i}}{\partial t}+b_{1} \frac{\partial \Phi}{\partial \rho}+b_{2} \lambda_{i}=C_{1} .
$$

The parallel momentum balance equation can be similarly written as

$$
a_{3} \frac{\partial}{\partial t} \frac{\partial \Phi}{\partial \rho}+a_{4} \frac{\partial \lambda_{i}}{\partial t}+b_{3} \frac{\partial \Phi}{\partial \rho}+b_{4} \lambda_{i}=C_{2} .
$$

Expressions for the constants $a, b$, and $C$ can be found in Ref. 12 , though they need to be slightly modified to account for the difference in angle domain and flux surface label between that reference and the current work. See Ref. 8 for the corrected expressions. Equations (19) and (20) can be written as

$$
A \frac{d \mathbf{X}}{d t}+B \mathbf{X}=C,
$$

with $\mathbf{X}=[\partial \Phi / \partial \rho ; \lambda]$, and $A, B$, and $C$ being vectors and arrays containing the constants noted above. With the definitions $D=A^{-1} B, S=A^{-1} C$, and $\Delta=\operatorname{det}(A)=a_{1} a-a_{2} a_{3}$, the system in Eq. (21) can be written as

$$
\frac{d \mathbf{X}}{d t}=d \mathbf{X}+S
$$

From this expression, it is possible to derive the eigenvalues of the system as

$$
\begin{aligned}
& \left.\begin{array}{l}
\gamma_{\mathrm{s}} \\
\gamma_{\mathrm{f}}
\end{array}\right\}=\frac{1}{2}\left(d_{11}+d_{22}\right) \\
& \pm \sqrt{\frac{1}{4}\left(d_{11}+d_{22}\right)^{2}-\left(d_{11} d_{22}-d_{12} d_{21}\right)} .
\end{aligned}
$$

This expression can be used to calculate the flow decay rates when the Hamada spectrum and basis vectors are known.

The two rates in Eq. (23) are the rates for the plasma flows and electric fields to decay, as can be shown as follows. Let the radial current be turned off at $t=t_{0}$, and call $\Phi_{0}^{\prime}=\partial \Phi / \partial \rho\left(t=t_{0}\right)$ the potential gradient when the electrode current is terminated and $\lambda_{0}=\lambda\left(t=t_{0}\right)$ the force free parallel flow at that time. The flow speeds at this time can be calculated from $\Phi_{0}^{\prime}$ and $\lambda_{0}$ using Eq. (10). The time evolution of the electric field and parallel flow can be written as

$$
\begin{aligned}
& \lambda(t)=N_{4} e^{\gamma_{\mathrm{s}}\left(t-t_{0}\right)}+N_{5} e^{\gamma_{\mathrm{f}}\left(t-t_{0}\right)}, \\
& \frac{\partial \Phi}{\partial \rho}(t)=N_{1} e^{\gamma_{\mathrm{s}}\left(t-t_{0}\right)}+N_{2} e^{\gamma\left(t-t_{0}\right)}+N_{3},
\end{aligned}
$$

where the following definitions have been used

$$
D_{1}=\left(\gamma_{\mathrm{f}}-d_{11}\right) S_{1}-d_{12} S_{2}, \quad D_{2}=d_{12} S_{2}-\left(\gamma_{\mathrm{s}}-d_{11}\right) S_{1},
$$

$$
\begin{aligned}
& D_{3}=\frac{1}{\left(\gamma_{\mathrm{f}}-\gamma_{\mathrm{s}}\right)}\left[\left(\gamma_{\mathrm{f}}-d_{11}\right) \Phi_{0}^{\prime}-d_{12} \lambda_{0}\right], \\
& D_{4}=\frac{1}{\left(\gamma_{\mathrm{f}}-\gamma_{\mathrm{s}}\right)}\left[d_{12} \lambda_{0}-\left(\gamma_{s}-d_{11}\right) \Phi_{0}^{\prime}\right],
\end{aligned}
$$

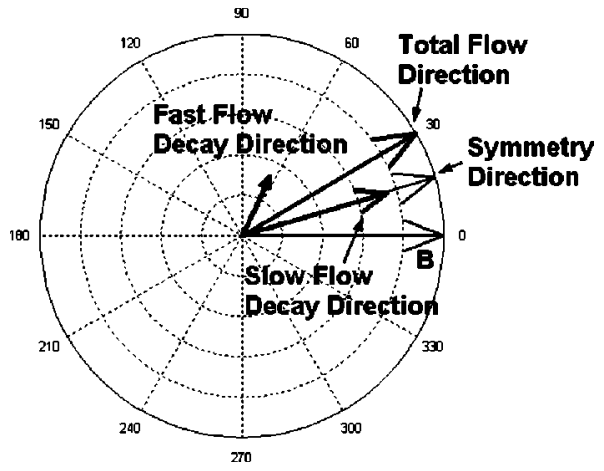

FIG. 10. Directions associated with the flow decay in the neoclassical formulation. The direction corresponding to the fast flow decay is the second term in parenthesis on the RHS of Eq. (28), while the slow flow decay direction is the first term.

$$
\begin{aligned}
& N_{1}=D_{3}+\frac{D_{1}}{\gamma_{\mathrm{s}}\left(\gamma_{\mathrm{f}}-\gamma_{\mathrm{s}}\right)}, \quad N_{2}=D_{4}+\frac{D_{2}}{\gamma_{\mathrm{f}}\left(\gamma_{\mathrm{f}}-\gamma_{\mathrm{s}}\right)}, \\
& N_{3}=\frac{\gamma_{\mathrm{f}} D_{1}+\gamma_{\mathrm{s}} D_{2}}{\gamma_{\mathrm{s}} \gamma_{\mathrm{f}}\left(\gamma_{\mathrm{f}}-\gamma_{\mathrm{s}}\right)}, \\
& N_{4}=\frac{D_{1}}{\gamma_{\mathrm{s}}} \frac{\gamma_{\mathrm{s}}-d_{11}}{d_{12}\left(\gamma_{\mathrm{f}}-\gamma_{\mathrm{s}}\right)}+\frac{D_{3}\left(\gamma_{\mathrm{s}}-d_{11}\right)}{d_{12}}, \\
& N_{5}=\frac{D_{2}}{\gamma_{\mathrm{f}}} \frac{\gamma_{\mathrm{f}}-d_{11}}{d_{12}\left(\gamma_{\mathrm{f}}-\gamma_{\mathrm{s}}\right)}+\frac{D_{4}\left(\gamma_{\mathrm{f}}-d_{11}\right)}{d_{12}} .
\end{aligned}
$$

The terms $S_{1}$ and $S_{2}$ are evaluated with $\left\langle\mathbf{J}_{\text {ext }} \cdot \nabla \rho>=0\right.$. With these definitions, the flow evolution at bias turn-off can be written as

$$
\begin{aligned}
\mathbf{U}\left(t>t_{0}\right)= & \left(N_{4} \mathbf{B}+\frac{c N_{1}}{B^{\zeta} \sqrt{g}} \mathbf{e}_{\alpha}\right) e^{\gamma_{\mathrm{s}}\left(t-t_{0}\right)} \\
& +\left(N_{5} \mathbf{B}+\frac{c N_{2}}{B^{\zeta} \sqrt{g}} \mathbf{e}_{\alpha}\right) e^{\gamma_{\mathrm{f}}\left(t-t_{0}\right)} .
\end{aligned}
$$

This expression illustrates that the neoclassical flow decay can be described by a two time-scale, two direction formulation. The flows decay in one direction at a rate $\gamma_{\mathrm{f}}$ and in a second direction at a rate $\gamma_{\mathrm{s}}$. With the knowledge of the Hamada basis vectors, one can use Eq. (28) to calculate the lab frame directions associated with the two decay rates.

The calculation shown in Fig. 10 illustrates these directions for the QHS configuration for the location of the low field side Mach probe. The plane of the figure is the toroidalpoloidal plane, and the coordinate system is rotated so that the magnetic field points directly to the right. The predicted direction of the total flow during bias points $\approx 30^{\circ}$ counterclockwise of horizontal in this figure. This direction is the vector sum of the flows in the fast and slow decay directions. The slow decay direction, corresponding to the first term in parenthesis on the right-hand side (RHS) of Eq. (28), is exactly parallel to the direction of symmetry in the $(n, m)$ $=(4,1)$ spectral component. The fast decay direction, corresponding to the second term in parenthesis on the RHS on Eq. (28), is not exactly perpendicular to the direction of sym- 
metry, because there are parallel flows associated with the flows across the direction of symmetry. The parameters in this calculation are similar to those in HSX except for the neutral density, which is increased by an order of magnitude compared to the experimental value. This increased neutral density (to $1 \times 10^{11} \mathrm{~cm}^{-3}$ in the calculation) has the effect of forcing more flow in the fast direction, causing the plot to be more clear than if the realistic neutral density were used. Also note that the length of the arrows has been normalized so that the total flow vector, the magnetic field vector $\mathbf{B}$, and symmetry direction vector all have unit length.

\section{The spin-up model}

To model the spin-up of the plasma flows and formation of the electric field, we have developed new modeling techniques based upon experimental observations. ${ }^{8}$ The data presented in Sec. III show that the electrode voltage is applied on a time-scale of $\sim 1 \mu \mathrm{s}$, and that the floating profile evolves on the same time-scale. Hence, to model the spin-up, we have developed a model where the initiating event is a quick change in the potential gradient:

$$
\frac{\partial \Phi}{\partial \rho}=\left\{\begin{array}{cc}
E_{\mathrm{r} 0}=\frac{-1}{e N_{i}} \frac{\partial \rho_{i}}{\partial \rho}, & t<0, \\
E_{\mathrm{r} 0}+\kappa_{\mathrm{E}}\left(1-e^{-t / \tau}\right), & t>0 .
\end{array}\right.
$$

In this expression, $\tau$ is a time on order of $1 \mu$ s. Since we are specifying the electric field evolution externally, the parallel momentum balance can be rewritten from Eq. (20) as

$$
a_{4} \frac{\partial \lambda_{i}}{\partial t}+b_{4} \lambda_{i}=C_{2}-a_{3} \frac{\partial}{\partial t} \frac{\partial \Phi}{\partial \rho}-b_{3} \frac{\partial \Phi}{\partial \rho} .
$$

Substituting the electric field evolution (29) into the parallel momentum balance (30) and solving the differential equation yields the time dependence of the bootstraplike part of the parallel flow.

$$
\lambda(t)=\kappa_{\mathrm{E}} Q_{1}\left[1-\left(1+Q_{2}\right) e^{-v_{F} t}+Q_{2} e^{-t / \tau}\right] .
$$

The constants are defined in terms of the viscous frequencies and the ion-neutral collision frequency as

$$
\begin{aligned}
& Q_{1}=-\frac{c}{\sqrt{g} B^{\alpha} B^{\zeta}}\left(\frac{t v_{\alpha}+v_{\text {in }} \frac{\left\langle\mathbf{B} \cdot \mathbf{B}_{\rho}\right\rangle}{\left\langle\boldsymbol{B}^{2}\right\rangle}}{t v_{\alpha}+v_{\zeta}+v_{\text {in }}}\right), \\
& Q_{2}=\left(\frac{t v_{\alpha}+v_{\zeta}+v_{\text {in }}}{t v_{\alpha}+v_{\text {in }} \frac{\left\langle\mathbf{B} \cdot \mathbf{B}_{\mathrm{p}}\right\rangle}{\left\langle\boldsymbol{B}^{2}\right\rangle}}\right)\left(\frac{t v_{\alpha}+\frac{\left\langle\mathbf{B} \cdot \mathbf{B}_{\rho}\right\rangle}{\left\langle\boldsymbol{B}^{2}\right\rangle}\left(v_{\text {in }}-\frac{1}{\tau}\right)}{t v_{\alpha}+v_{\zeta}+v_{\text {in }}-\frac{1}{\tau}}\right), \\
& v_{F}=\frac{b_{4}}{a_{4}} t v_{\alpha}+v_{\zeta}+v_{\text {in }} .
\end{aligned}
$$

The quantity $Q_{2}$ is much less than one for HSX conditions. With this determination of the $\lambda$ and electric field evolution, the total flow speed evolution at bias turn-on can be calculated as

$$
\begin{aligned}
\mathbf{U}(t)= & U_{\mathrm{E}}^{\alpha}\left(1-e^{-t / \tau}\right) \mathbf{e}_{\alpha}+\mathbf{B} Q_{1} \kappa_{\mathrm{E}}\left[1-\left(1+Q_{2}\right) e^{-v_{\mathrm{F}} \mathrm{t}}\right. \\
& \left.+Q_{2} e^{-\mathrm{t} / \tau}\right],
\end{aligned}
$$

where

$$
U_{\mathrm{E}}^{\alpha}=\frac{c}{B^{\zeta} \sqrt{g}} \kappa_{\mathrm{E}} .
$$

Equation (34) illustrates that there are two time-scales for the flow to evolve in this model. The $\mathbf{E} \times \mathbf{B}$ and PfirschSchlueter flows are encapsulated in the first term on the RHS of Eq. (34) and grow on the externally imposed time-scale. The bootstrap like parallel part of the flow grows on the time-scale $\nu_{\mathrm{F}}$. Note that this second time-scale is solely determined by the plasma parameters and magnetic geometry.

The final constraint in this model is provided by poloidal momentum balance, given by Eq. (19). The electric field and both components of the flow velocity have now been specified. The remaining free parameter in the poloidal momentum balance is the external current, which can be calculated as

$$
\left\langle\mathbf{J}_{\text {ext }} \cdot \nabla \rho\right\rangle=Q_{3} e^{-\mathrm{t} / \tau}+Q_{4} e^{-v_{\mathrm{F}} \mathrm{t}}+Q_{5} .
$$

The constants in this equation are given by

$$
\begin{aligned}
Q_{3}= & m_{\mathrm{i}} N_{\mathrm{i}}\left\langle B_{\mathrm{P}}^{2}\right\rangle\left(\frac{c}{\sqrt{g} B^{\alpha} B^{\zeta}}\right)^{2} \kappa_{\mathrm{E}}\left(a_{1} \frac{1}{\tau}-Q_{1} Q_{2} \frac{a_{2}}{\tau}\right. \\
& \left.+b_{2} Q_{1} Q_{2}-b_{1}\right), \\
Q_{4}= & m_{\mathrm{i}} N_{\mathrm{i}}\left\langle B_{\mathrm{P}}^{2}\right\rangle\left(\frac{c}{\sqrt{g} B^{\alpha} B^{\zeta}}\right)^{2} \kappa_{\mathrm{E}}\left[a_{2} Q_{1} v_{\mathrm{F}}\left(1+Q_{2}\right)\right. \\
& \left.-b_{2} Q_{1}\left(1+Q_{2}\right)\right], \\
Q_{5}= & m_{\mathrm{i}} N_{\mathrm{i}}\left\langle B_{\mathrm{P}}^{2}\right\rangle\left(\frac{c}{\sqrt{g} B^{\alpha} B^{\zeta}}\right)^{2} \kappa_{\mathrm{E}}\left(b_{1}+b_{2} Q_{1}\right) .
\end{aligned}
$$

\section{Comparison of the models}

The modeling has now predicted three important timescales: the fast $\left(\gamma_{\mathrm{f}}\right)$ and slow $\left(\gamma_{\mathrm{s}}\right)$ rates for the flow and electric field decay, and the rate $\nu_{\mathrm{F}}$ for the parallel flows to rise. These time-scales are plotted as a function of minor radius for the QHS and mirror configurations in Fig. 11. The plasma parameters in the calculations are similar to those in HSX, except that the neutral atom density has been set to zero. Eliminating ion-neutral collisions emphasizes the differences in neoclassical parallel viscosity between the configurations.

The neoclassical slow rate illustrates the largest difference between the QHS and mirror configurations. In the plasma core, the difference is about two orders of magnitude, decreasing to a factor of 10 closer to the edge. The fast rates in the QHS and mirror configuration are closer to each other, and differ by only about $30 \%$ across the entire minor radius. Hence, the neoclassical expectation is that the fast time-scale for flow decay will be similar between the two configurations, but that the slower decay time-scales will show a large 


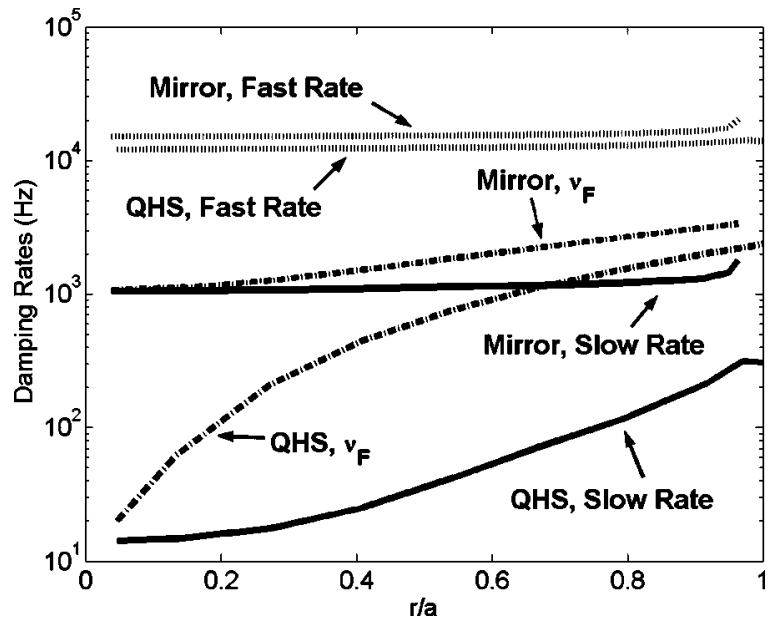

FIG. 11. The three neoclassical damping rates for the mirror and QHS configurations, as a function of minor radius. The neutral density has been set to zero in this calculation to emphasize the differences in parallel viscosity between the configurations.

difference. The difference in $\nu_{\mathrm{F}}$ between the QHS and mirror configurations is smaller than the difference in the slow rates.

For both the QHS and mirror configurations, the hybrid time-scale $\nu_{\mathrm{F}}$ resides between the fast and slow decay timescales. This can be understood as follows. There are two variables necessary to describe the evolution of the flows on the two-dimensional magnetic surface. These variables could be any two of the toroidal flow, poloidal flow, parallel flow, or radial electric field; ${ }^{40}$ we have chosen the electric field and parallel flows in Eqs. (19) and (20). For the decay model, the driving term [the radial current on the RHS of Eq. (19)] is changed, and the system responds with its natural frequencies $\left(\gamma_{\mathrm{f}}\right.$ and $\left.\gamma_{\mathrm{s}}\right)$. When the electric field is changed on some externally imposed time-scale, as in the spin-up model, one of the system variables is externally forced. This leaves only one remaining time-scale. This time-scale then has information about both of the natural time-scales $\left(\gamma_{\mathrm{f}}\right.$ and $\left.\gamma_{\mathrm{s}}\right)$ and is intermediate to them.

It should be noted that in addition to the mirror field, the introduction of magnetic islands into the configuration can lead to symmetry breaking and an increase in viscous damping. This damping mechanism is discussed in Ref. 41, and will not be considered further in this paper.

\section{COMPARISON BETWEEN MEASUREMENTS AND NEOCLASSICAL MODELING}

In comparing the modeling with the measurements, the topic naturally divides itself into three areas. Section V A will describe comparisons between the neoclassical model and the steady state radial conductivity and flow direction. Section V B will compare the spin-up model to measurements. A comparison of the measured relaxation of the flows and potentials with the neoclassical relaxation model is given in Sec. V C. Section V D provides a discussion of the observed enhanced flow damping in HSX. Note that for these theory/experiment comparisons, the toroidal flux $\psi$ is used as a magnetic surface label.

\section{A. The steady state radial conductivity and flow direction}

The relationship between the steady state radial current and the radial electric field is determined by the radial conductivity as described by Eq. (15) . It appears that before the electrode is energized, the externally driven radial current would be zero, implying that the plasma radial current is zero and

$$
\left\langle\mathbf{E}_{\mathrm{r}} \cdot \nabla \psi\right\rangle=\frac{\left\langle\nabla \mathrm{p}_{i} \cdot \nabla \psi\right\rangle}{e N_{\mathrm{i}}} .
$$

The pressure gradient balances the electric field, and there is no plasma flow. Note that inclusion of temperature gradient effects in the viscosities would modify this expression, $8,35,42$ allowing for flow with no external radial current. It is not anticipated that these corrections are important for the low density ECH plasmas described in this article, where cool ions and small ion temperature gradients are inferred from Doppler spectroscopy. ${ }^{8}$

Contrary to the expectations from Eq. (38), measurements show that for most unbiased plasmas in HSX, both the pressure gradient and the floating potential increase towards the core. Langmuir probe and Thomson scattering indicate that the $T_{\mathrm{e}}$ profile is peaked in the core, with only a small slope in the outer half radius (with $T_{\mathrm{e}}$ increasing toward the magnetic axis). Hence, we infer that both the plasma potential and the ion pressure increase towards the core. This is apparently in contradiction of the prediction of Eq. (38).

While the reason for this contradiction is not at present clear, it is hypothesized that there is some radial current driven before the electrode bias is applied. The positive potentials before bias (see Fig. 6) would correspond to some non-ambipolar mechanism driving electrons from HSX. This mechanism would take the place of the biased electrode drawing electrons out of the plasma, and a return current would need to flow to maintain ambipolarity. Possible mechanisms for this electron flux include convective fluxes due to the $\mathrm{ECH}^{43}$ or nonambipolar turbulent fluxes. ${ }^{44}$

Assuming for the moment that a radial current is indeed flowing before the electrode voltage is applied, then Eq. (15) before bias becomes

$$
\begin{aligned}
\frac{\langle|\nabla \psi|\rangle}{A_{\text {surf }}} I_{\text {pre-bias }}= & \sigma_{\perp}\left[\left(\left.\frac{d \mathrm{~V}_{\mathrm{f}}}{d \psi}\right|_{\text {pre-bias }}+\kappa \frac{d T_{e}}{d \psi}\right)\langle\nabla \psi \cdot \nabla \psi\rangle\right. \\
& \left.+\frac{\left\langle\nabla p_{\mathrm{i}} \cdot \nabla \psi\right\rangle}{e N_{\mathrm{i}}}\right]
\end{aligned}
$$

where we have used $\langle\mathbf{J} \cdot \nabla \psi\rangle=I\langle\nabla \psi\rangle / A_{\text {surf }}$ and $\Phi_{\mathrm{P}}=V_{\mathrm{f}}+\kappa T_{\mathrm{e}}$. In these expressions, $A_{\text {surf }}$ is the area of the magnetic surface, $\Phi_{\mathrm{P}}$ is the plasma potential, and $\kappa$ is a species dependent constant. During the bias steady state, the total current flowing through the plasma is given by $I_{\text {total }}=I_{\text {electrode }}+I_{\text {pre-bias }}$. This leads to the version of Eq. (15) during bias as 


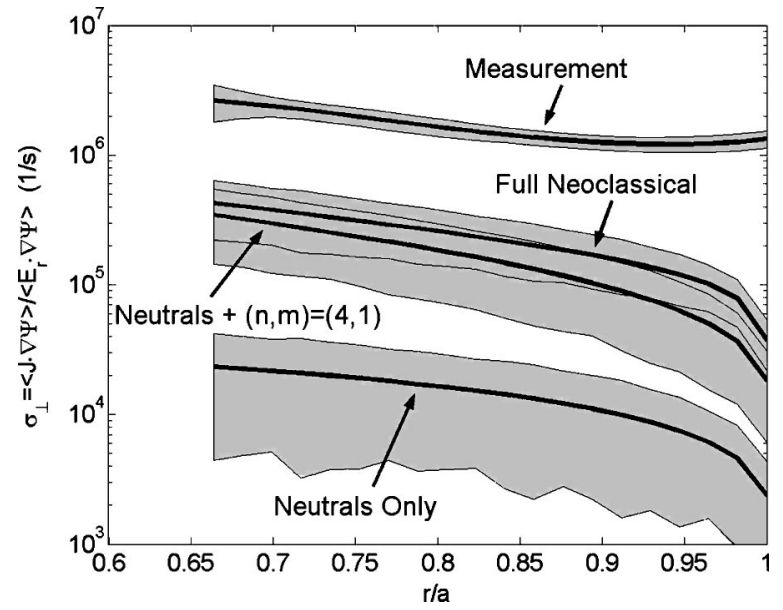

FIG. 12. The measured radial conductivity profiles in the QHS configuration with line average density of $1 \times 10^{12} \mathrm{~cm}^{-3}$. Neoclassical predictions are also shown corresponding to only neutrals, neutrals and the $(n, m)=(4,1)$ spectral component, and the combination of neutrals and all magnetic field ripples.

$$
\begin{aligned}
\frac{\langle|\nabla \psi|\rangle}{A_{\text {surf }}} I_{\text {total }}= & \sigma_{\perp}\left[\left(\left.\frac{d V_{\mathrm{f}}}{d \psi}\right|_{\text {during-bias }}+\kappa \frac{d T_{\mathrm{e}}}{\mathrm{d} \psi}\right)\langle\nabla \psi \cdot \nabla \psi\rangle\right. \\
& \left.+\frac{\left\langle\nabla p_{\mathrm{i}} \cdot \nabla \psi\right\rangle}{e N_{\mathrm{i}}}\right],
\end{aligned}
$$

where it has been assumed that the electron temperature and ion pressure gradients do not change when the bias is applied. Subtraction of Eq. (39) from Eq. (40) and solving for the radial conductivity yields

$$
\sigma_{\perp}=\frac{\langle|\boldsymbol{\nabla} \psi|\rangle}{\langle\boldsymbol{\nabla} \psi \cdot \boldsymbol{\nabla} \psi\rangle A_{\text {surf }}} \frac{I_{\text {electrode }}}{\frac{d}{d \psi}\left(\left.V_{\mathrm{f}}\right|_{\text {during-bias }}-\left.V_{\mathrm{f}}\right|_{\text {pre-bias }}\right)} .
$$

The terms $\langle\nabla \psi \cdot \nabla \psi\rangle,\langle|\nabla \psi|\rangle$, and $A_{\text {surf }}$ are calculated for many surfaces as part of the Hamada basis vector calculation.

A comparison of the measured and modeled radial conductivity in the QHS configuration is illustrated in Fig. 12. The line average plasma density in these discharges in 1 $\times 10^{12} \mathrm{~cm}^{-3}$, and the neutral density is $\sim 1 \times 10^{10} \mathrm{~cm}^{-3}$. The measured radial conductivity is the top curve (note that conductivity has units of $1 / s$ in cgs units). There are three neoclassical predictions shown in the figure. The bottom curve is the radial conductivity with all of the viscous frequencies in Eq. (16) set to zero; only neutrals are considered in this calculation. The next higher curve shows the radial conductivity considering neutrals and the $(n, m)=(4,1)$ spectral component. The addition of the single spectral component causes a significant increase in the radial conductivity, as has been noted previously for the tokamak case. ${ }^{11}$ The uppermost neoclassical prediction shows the radial conductivity including neutrals and all magnetic field ripples. The error regions in these and subsequent calculations are based upon Monte Carlo propagation of the estimated errors in the neutral density, plasma density, and ion temperature through the formulas presented in Sec. IV. The difference between the measured and neoclassically predicted radial conductivity is

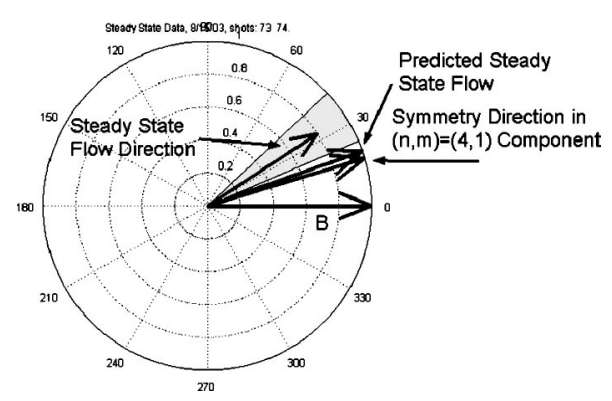

FIG. 13. Comparison of the predicted and measured steady state flow directions, on the low field side in the QHS configuration. The coordinate system is orientated so that the magnetic field points directly to the right, and the direction of symmetry in the $(n, m)=(4,1)$ spectral component is shown as well.

approximately a factor of 10 , leading to the conclusion that the radial conductivity is anomalous. Note that because the floating potential profiles on the high and low field sides are very similar when the radial coordinate is mapped to toroidal flux, the radial conductivity determined from the two locations would be similar.

This anomalously large radial conductivity is seen in toroidally symmetric devices. ${ }^{45}$ Rozhansky and Tendler ${ }^{46}$ have constructed a model that approximately predicts the radial conductivity in the $L$ mode in TUMAN-3. This model assumes that there exists anomalous shear viscosity to damp the toroidal flow. Under the assumption that gradients in the poloidal flow are small, the radial conductivity can be expressed as

$$
\sigma_{\perp}=-\frac{c^{2} m_{\mathrm{i}} N_{\mathrm{i}} g \sqrt{\pi} v_{\mathrm{ti}}}{2 R_{\mathrm{o}} B_{\mathrm{o}}^{2}} .
$$

When evaluated for the parameters of these QHS discharges, the prediction of Eq. (42) is $\sigma_{\perp} \sim 1 \times 10^{6} \mathrm{~s}^{-1}$, which is approximately the experimental value presented above.

Similar measurements have been made in the mirror configuration on HSX. The measured radial conductivity is $\approx 30 \%$ higher in the mirror configuration than the QHS. The neoclassical prediction is a factor of $\sim 4-5$ larger than the prediction for the QHS case. Hence, the theory/experiment is closer in the mirror case than the QHS, but is still off by a factor of $\sim 3$.

In addition to the steady state radial conductivity, the neoclassical modeling also predicts the direction of net plasma flow, via Eqs. (17) and (18). With our knowledge of the Hamada basis vectors in the laboratory frame, we are able to make comparisons between the measured flow direction and the neoclassical prediction. An example comparison is shown in Fig. 13, for a measurement taken with the low field side probe $1 \mathrm{~cm}$ inside the separatrix. The line-average density in this QHS discharge was $1 \times 10^{12} \mathrm{~cm}^{-3}$, and the neutral density is estimated to be $\sim 1 \times 10^{10} \mathrm{~cm}^{-3}$.

This figure is in the same coordinate system as Fig. 10, with the magnetic field pointing directly to the right and with the direction of symmetry rotated counterclockwise of the magnetic field. The predicted steady state flow direction is rotated slightly counterclockwise from the symmetry direction, and the measured steady state flow is rotated farther 


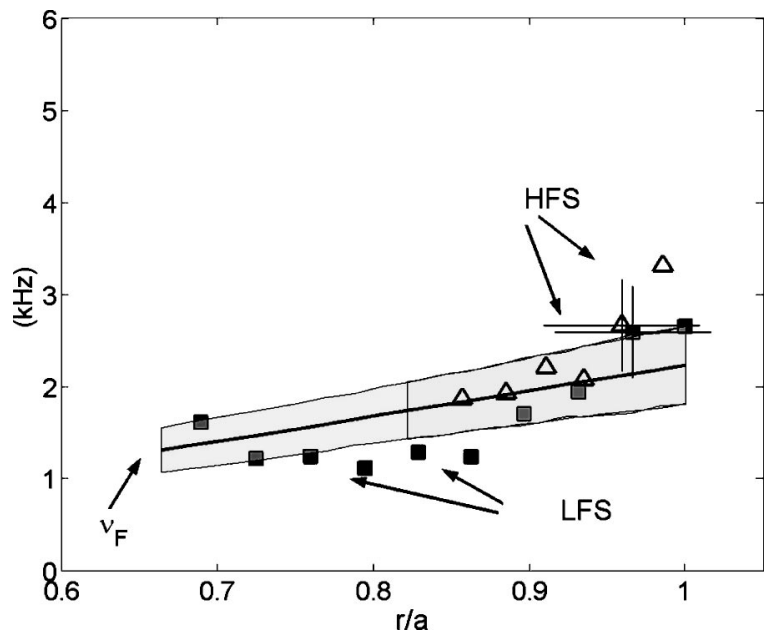

FIG. 14. Comparison of the slow flow rise rates $\left[r_{\mathrm{s}}\right.$, in Eq. (7)], as measured in the QHS configuration in the high and low $|B|$ regions. The neoclassical rise rate $\nu_{\mathrm{F}}$ calculated from Eq. (33) is shown for comparison.

counterclockwise. We conclude that the measured flow is rotated away from the symmetry direction compared to the neoclassical prediction. The subject of the flow direction will be revisited in Sec. V D.

\section{B. The measured and modeled spin-up of the plasma flows}

Consider now the spin-up of the plasma when the electric field is applied. The measurements illustrated in Fig. 7 show that the electric field is formed extremely quickly when the bias is applied. This is implicitly consistent with the model described in Sec. IV C, and was the observation that motivated the model in the first place. It is then important to compare the measured and modeled evolution of the plasma flows.

The rate of slow flow rise $\left[r_{\mathrm{s}}\right.$, from the fits of Eq. (7)] is shown in Fig. 14, where the two symbols illustrate measurements taken on the low and high field sides of HSX. These discharges have a line average density of $1 \times 10^{12} \mathrm{~cm}^{3}$ and an ion temperature of $20 \mathrm{eV}$. The physical locations of the tips of the two probes have been mapped to toroidal flux so that they can be compared on the same axis. There is excellent agreement between the measured slow rise rates on the low and high field sides, illustrating that this time-scale is an appropriate global quantity for comparison with modeling. The modeled time-scale $\nu_{\mathrm{F}}$ is plotted on the data as well. A neutral density of $1 \times 10^{10} \mathrm{~cm}^{-3}$ is used in the calculation based on the $H_{\alpha}$ measurements and DEGAS simulations. The measured time-scales show good agreement with the predicted time-scale based upon neoclassical modeling, both in numerical value and in the radial profile.

Measurements have been made where the biasing electrode and Mach probes are held at a fixed location while the plasma density is scanned via gas puffing. The data shows that the slow rise rate is largely independent of density in both the QHS and mirror configurations. Note that the timescale $\nu_{\mathrm{F}}$ is also independent of density in the plateau regime, consistent with the measured scaling.

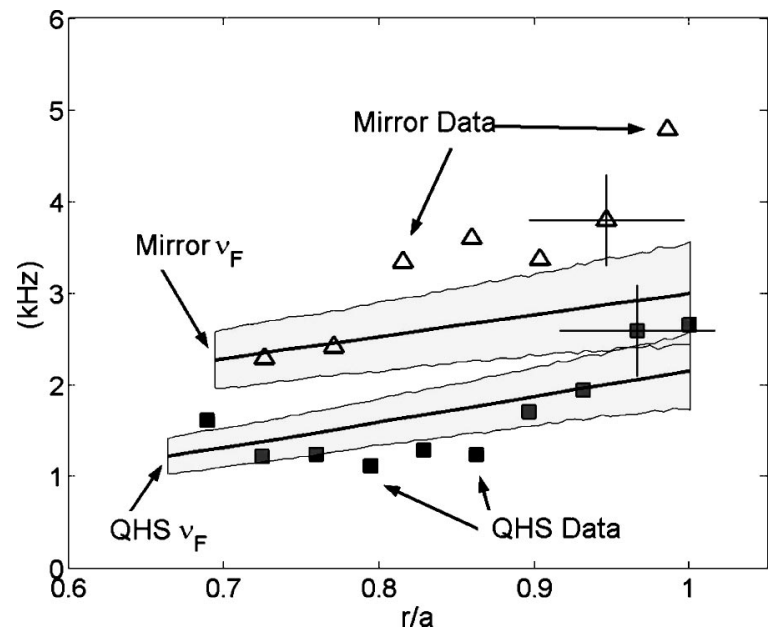

FIG. 15. Comparison of the slow flow rise rates in the QHS and mirror configurations. The neoclassical rise rate $\nu_{\mathrm{F}}$ from Eq. (33) is illustrated in the figure, and agrees with the data in both configurations.

These measurements have been made in the QHS and mirror configurations of HSX at the same input power $(50 \mathrm{~kW})$, line average density $\left(1 \times 10^{12} \mathrm{~cm}^{-3}\right)$, and field strength $(B=0.5 \mathrm{~T})$. The measured impurity ion temperature of $\sim 20 \mathrm{eV}$ is similar in the two configurations. The measured slow rise rates $r_{\mathrm{s}}$ for the QHS and mirror configurations are shown in Fig. 15. The measurements are made with the low field side Mach probe in both cases. The measurements in the QHS configuration show a reduced rise rate compared to the mirror configuration, as expected for a configuration with reduced viscous damping. The calculated rates $\nu_{\mathrm{F}}$ are illustrated in the figure as well, and show good agreement with the data. Note that the difference in the modeled predictions is due to neoclassical viscous damping. We thus infer that the measured reduction in damping in the quasisymmetric case is also due to reduced neoclassical viscosity.

\section{The relaxation of the floating potential and plasma flows after bias termination}

We will next consider the dynamics when the electrode current is broken. We typically fit the decay of the electrode current and voltage to a single time constant exponential,

$$
f=\left\{\begin{array}{cc}
f_{\mathrm{o}}, & t<t_{\mathrm{o}} \\
\left(f_{\mathrm{o}}-f_{\mathrm{ss}}\right) \exp \left[-r\left(t-t_{\mathrm{o}}\right)\right]+f_{\mathrm{ss},} & t>t_{\mathrm{o}},
\end{array}\right.
$$

where $f$ is either the electrode current or voltage, $f_{\mathrm{o}}$ is the value before the bias pulse is terminated at $t=t_{\mathrm{o}}$, and $f_{\mathrm{ss}}$ is the value long after the decay has finished. In Fig. 16, we plot the decay rate of the electrode voltage and current as a function of the line average plasma density for QHS plasmas with the bias probe located at $r / a=0.65$. The current decay rate is typically $10^{6} \mathrm{~s}^{-1}$, satisfying the model requirement that the current termination be the fast initiating event. The electrode voltage typically decays at a rate of $\sim 30-50 \mathrm{kHz}$.

Also shown in the graphs is the neoclassical fast decay rate $\gamma_{\mathrm{f}}$ at the location of the electrode, as a function of plasma density. The current termination occurs substantially 


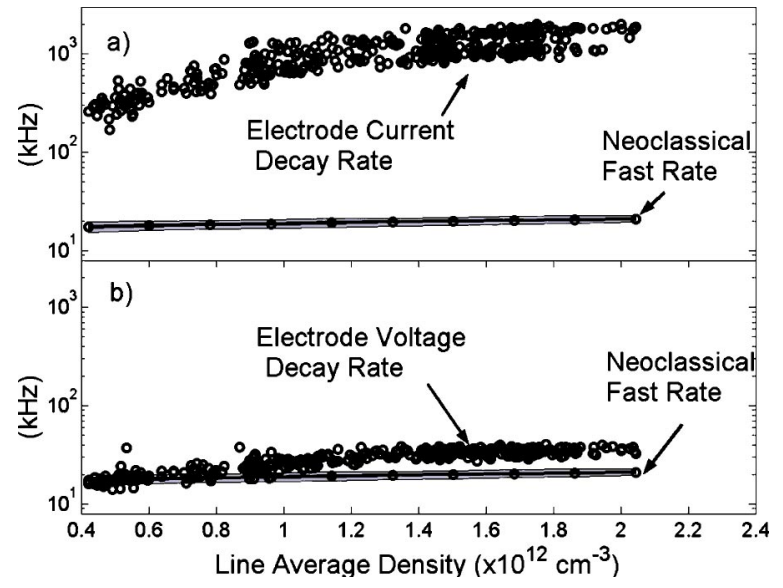

FIG. 16. The decay rate $[r$ in Eq. (43)] for (a) the electrode current and (b) the electrode voltage. The fast neoclassical damping rate $\left(\gamma_{\mathrm{f}}\right)$ from Eq. (23) is also shown.

faster than the fast neoclassical rate, but the electrode voltage decay rate is within a factor of 2 of the neoclassical rate. This comparison should be taken with caution, though, as the neoclassical viscosities in the modeling are not necessarily accurate on this fast time-scale (see discussion in Sec. IV A).

Considering the relaxation of the plasma flows, it was observed above that one component of the flows tends to decay on the same fast time-scale as the floating potential. The slowly decaying component of the flow is mainly in the parallel direction, in contrast with the neoclassical prediction that the slowly decaying component of the flow should be in the symmetry direction. The rates for these flows to decay $\left(r_{s}\right)$ are illustrated in Fig. 17, for the QHS and mirror configurations of HSX. The flows decay more slowly in the quasisymmetric configuration, as anticipated for the configuration with reduced damping. The difference in measured damping rates is $\sim(2-3) \times 10^{3} \mathrm{~s}^{-1}$.

The neoclassical slow rate $\gamma_{\mathrm{s}}$ is illustrated in the figure, for each of the two configurations. Recall that this rate corresponds neoclassically to the rate at which flows in the di-

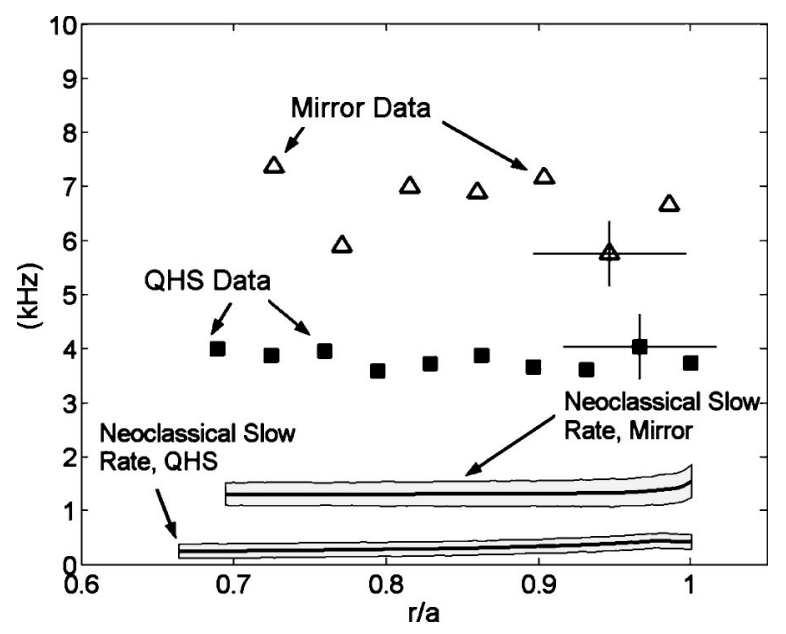

FIG. 17. The decay rate for the slow component of the flow to decay for the QHS and mirror configuration. The neoclassical slow flow decay rate $\left(\gamma_{\mathrm{s}}\right)$ from Eq. (23) is plotted for comparison. rection of symmetry decay. For the QHS configuration, this rate is dominated by ion-neutral collisions, and is approximately given by $v_{\text {in }} \approx n_{\mathrm{n}} 10^{-8} T_{\mathrm{i}}^{318}=150-300 \mathrm{~s}^{-1}$. Hence, the rate at which the flows are damped is not consistent with neoclassical theory; the measurement and prediction differ by a factor of $\approx 10$.

The measured mirror slow damping rates also differ from the predicted neoclassical slow damping rates, although the ratio of measurement to neoclassical prediction is closer than in the QHS case. Note that the difference between the measurements of $\sim 1.5 \times 10^{3} \mathrm{~s}^{-1}$ is similar to the difference in the neoclassical predictions. In this sense, we hypothesize that there is an additional source of damping which diminishes the predicted neoclassical difference. Nevertheless, the results show that the damping of flows is reduced with quasisymmetry, even in the presence of the anomalous flow damping.

\section{Discussion of enhanced flow damping in HSX}

The results presented above provide evidence that the faster time-scales $\left(\gamma_{\mathrm{f}}\right.$ and $\nu_{\mathrm{F}}$, see caveat about fast timescales in Sec. IV A) are approximately described by neoclassical theory for these measurements in HSX, but that the slower damping time-scale and radial conductivity are not. This result is not a surprise: many tokamak experiments have documented that the damping of flows in the symmetry direction (the toroidal direction in that case) is faster than neoclassical theory predicts. ${ }^{45,47-52}$ In this sense, the damping of flows in the quasisymmetric HSX is similar to that in axisymmetric tokamaks.

To further test this hypothesis, we have made comparisons between the measurements and calculations where the neutral density has been artificially increased to mimic extra flow damping. For the comparisons presented below, the neutral density has been increased from its measured value of $1 \times 10^{10} \mathrm{~cm}^{-3}$ to a value of $1.3 \times 10^{11} \mathrm{~cm}^{-3}$. The ionneutral collision frequency in this case is increased to $\nu_{\text {in }}$ $=33001 / \mathrm{s}$. There is no reason to believe that the neutral density in HSX is this large. In this sense, the much increased $\nu_{\text {in }}$ would be more accurately thought of as $\nu_{\text {eff }}$, an effective rate for momentum loss from the system.

Figure 18 presents a modeling/experiment comparison for the artificially large neutral density. The top frame shows the slow flow decay rate for the QHS configuration, where measurements from the high and low field side Mach probes are displayed. As noted with respect to the slow flow rise and floating potential decay rates, the rates measured at the two locations agree well with each other. With the artificially high neutral density given above, the agreement with the modeling is excellent. The bottom frame shows the radial conductivity measurement and neoclassical prediction (including all spectral components and artificially increased neutral density) for the same neutral density. The agreement is substantially improved across most of the measurement region.

The steady state flow direction comparison is shown in Fig. 19, where the neoclassical prediction is calculated using the artificially large neutral density. Compared to Fig. 13, the 

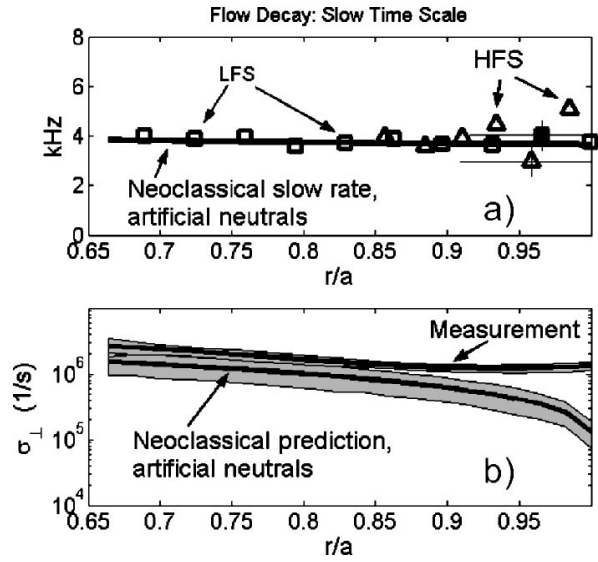

FIG. 18. (a) The decay rate for the slow component of the flow, compared to the neoclassical theory in Eq. (23) with artificially increased neutral damping, and (b) the measured radial conductivity compared to the prediction of Eq. (16) with the increased neutral damping.

agreement between the prediction and the measurements are much improved when the damping is increased. In particular, the predicted flow direction is rotated away from the symmetry direction by the same amount as the measurement.

While this artificially large neutral density improves all the comparisons noted in this section, it tends to degrade the agreement with the spin-up model. A more appropriate means of formulating the enhanced flow damping, probably including some anomalous shear viscosity, appears to be necessary to further understand the flow damping in HSX.

\section{SUMMARY}

In summary, our measurements indicate a distinct asymmetry in plasma parameter evolution between the spin-up and the spin-down. This asymmetry is due to the different initiating events; the electric field application initiates the spin-up, while open-circuiting the electrode current initiates the spin-down. Two time-scales are observed in the flow evolution, both during spin-up and spin-down, and techniques have been developed to extract these time-scales from the measurements. The time-scales for the flow to rise are in reasonable agreement with a model where the electric field formation initiates the spin-up of the plasma flows. The time to complete the spin-up is longer in the quasisymmetric configuration of HSX than in the symmetry broken configuration, in agreement with neoclassical modeling. The slower

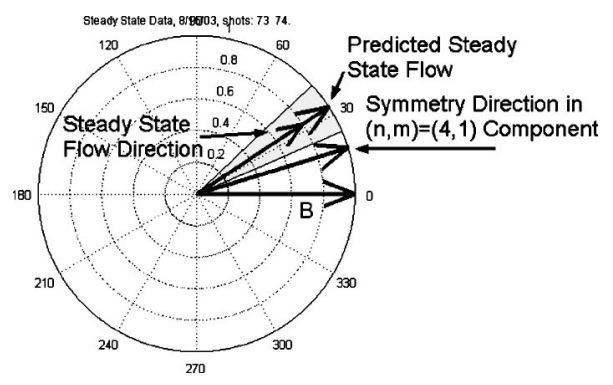

FIG. 19. Comparison between the measured and modeled plasma flow directions, where the prediction is based on Eq. (17) with the artificially large neutral damping. time-scale for the flow to decay is longer in the QHS case than the mirror, consistent with our neoclassical expectations. However, this time-scale is significantly shorter than the neoclassically predicted time-scale.

\section{ACKNOWLEDGMENTS}

The authors gratefully acknowledge the advice, assistance, and encouragement of A. F. Almagri, F. S. B. Anderson, C. Deng, W. Guttenfelder, C. Hegna, K. Likin, P. Probert, and K. C. Shaing. This research benefited greatly from the expertise of the HSX technical staff, including A. Piccione, M. Frankowski, E. Jolitz, and L. K. Neisius. This research was funded by the United States Department of Energy.

${ }^{1}$ J. Nuhrenberg and R. Zille, Phys. Lett. 114, 129 (1986).

${ }^{2}$ J. Nuhrenberg and R. Zille, Phys. Lett. A 129, 113 (1988).

${ }^{3}$ K. H. Burrell, Phys. Plasmas 4, 1499 (1997).

${ }^{4}$ M. C. Zarnstorff, L. A. Berry, A. Brooks, E. Fredrickson, G-Y. Fu, S. Hirshman, S. Hudson, L-P. Ku, E. Lazarus, D. Mikkelsen, D. Monticello, G. H. Neilson, N. Pomphrey, A. Reiman, D. Spong, D. Strickler, A. Boozer, W. A. Cooper, R. Goldston, R. Hatcher, M. Isaev, C. Kessel, J. Lewandowski, J. F. Lyon, P. Merkel, H. Mynick, B. E. Nelson, C. Neuhrenberg, M. Redi, W. Reiersen, P. Rutherford, R. Sanchez, J. Schmidt, and R. B. White, Plasma Phys. Controlled Fusion 43, 237 (2001).

${ }^{5}$ S. Okamura, K. Matsuoka, S. Nishimura, M. Isobe, I. Nomura, C. Suzuki, A. Shimizu, S. Murakami, N. Nakajima, M. Yokoyama, A. Fujisawa, K. Ida, K. Itoh, P. Merkel, M. Drevlak, R. Zille, S. Gori, and J. Nuhrenberg, Nucl. Fusion 41, 1865 (2001).

${ }^{6}$ D. A. Spong, S. P. Hirshman, L. A. Berry, J. F. Lyon, R. H. Fowler, D. J. Strickler, M. J. Cole, B. N. Nelson, D. E. Williamson, A. S. Ware, D. Alban, R. Sanchez, G. Y. Fu, D. A. Monticello, W. H. Miner, and P. M. Valanju, Nucl. Fusion 41, 711 (2001).

${ }^{7}$ F. S.B. Anderson, A. F. Almagri, D. T. Anderson, P. G. Mathews, J. N. Talmadge, and J. L. Shohet, Fusion Technol. 27, 273 (1995).

${ }^{8}$ S. P. Gerhardt, Ph.D. thesis, University of Wisconsin-Madison, 2004.

${ }^{9}$ K. Ida and N. Nakajima, Phys. Plasmas 4, 310 (1997).

${ }^{10}$ K. Ida, Y. Mjura, K. Kondo, J. V. Hofmann, F. Sano, S. Hidekuma, H. Yamada, H. Iguchi, T. Matsuda, H. Zushi, T. Obiki, K. Watanabe, J. Geiger, N. Nakajima, and F. Rau, in International Conference on Plasma Physics ICPP 1994, edited by Paulo H. Sakanaka and Michael Tendler, AIP Conf. Proc. No. 345 (AIP, Woodbury, NY, 1995).

${ }^{11}$ J. N. Talmadge, B. J. Peterson, D. T. Anderson, F. S. B. Anderson, H. Dahi, J. L. Shohet, M. Coronado, K. C. Shaing, M. Yokoyama, M. Wakatani, Proceedings of the 15th International Conference on Plasma Physics and Controlled Fusion Research, Seville, 1994 (IAEA, Vienna, 1995), Vol. 1, p. 797.

${ }^{12}$ M. Coronado and J. N. Talmadge, Phys. Fluids B 5, 1200 (1993).

${ }^{13}$ R. R. Weynants, G. Van Oost, G. Bertschinger, J. Boedo, P. Brys, T. Delvigne, K. H. Dippel, F. Durodie, H. Euringer, K. H. Finken, D. S. Gray, J. D. Hey, D. L. Hillis, J. T. Hogan, L. Konen, R. Leners, A. M. Messiaen, A. Pospieszczyck, U. Samm, R. P. Schorn, B. Schweer, G. Telesca, R. Van Nieuwenhove, and P. E. Vandenplas, Nucl. Fusion 32, 837 (1992).

${ }^{14}$ R. J. Taylor, M. L. Brown, B. D. Fried, H. Grote, J. R. Liberati, G. J. Morales, P. Pribyl, D. Darrow, and M. Ono, Phys. Rev. Lett. 63, 2365 (1989).

${ }^{15}$ S. P. Gerhardt, D. T. Anderson, F. S. B. Anderson, and J. N. Talmadge, Rev. Sci. Instrum. 75, 4621 (2004).

${ }^{16}$ C. S. MacLatchy, C. Boucher, D. A. Poirier, and J. Gunn, Rev. Sci. Instrum. 63, 3923 (1992).

${ }^{17}$ R. Back and R. D. Bengston, Rev. Sci. Instrum. 68, 377 (1997).

${ }^{18}$ B. J. Peterson, J. N. Talmadge, D. T. Anderson, F. S. B. Anderson, and J. L. Shohet, Rev. Sci. Instrum. 65, 2599 (1994).

${ }^{19}$ I. H. Hutchinson, Plasma Phys. Controlled Fusion 44, 1953 (2002).

${ }^{20}$ S. P. Gerhardt, J. M. Canik, D. T. Anderson, and L. Owen, Rev. Sci. Instrum. 75, 2981 (2004).

${ }^{21}$ D. B. Heifetz, D. Post, M. Petravic, J. Weisheit, and G. Bateman, J. Comput. Phys. 46, 309 (1982).

${ }^{22}$ C. Deng, D. L. Brower, W. X. Ding, A. F. Almagri, D. T. Anderson, F. S. 
B. Anderson, S. P. Gerhardt, P. Probert, and J. N. Talmadge, Rev. Sci. Instrum. 74, 1625 (2003).

${ }^{23}$ W. D. D'haeseleer, W. N.G. Hitchon, J. D. Callen, and J. L. Shohet, Flux Coordinates and Magnetic Field Structure (Springer, Berlin, 1991).

${ }^{24}$ C. Hidalgo, M. A. Pedrosa, N. Dreval, K. J. McCarthy, L. Eliseev, M. A. Ochando, T. Estrada, I. Pastor, E. Ascasibar, E. Calderon, A. Cappa, A. A. Chmyga, A. Fernandez, B. Goncalves, J. Herranz, J. A. Jimenez, S. M. Khrebtov, A. D. Komarov, A. S. Kozachok, L. Krupnik, A. LopezFraguas, A. Lopez-Sanchez, A. V. Melnikov, F. Medina, B. vanMilligen, C. Silva, F. Tabares, and D. Tafalla, Plasma Phys. Controlled Fusion 46, 287 (2004)

${ }^{25}$ G. S. Kirnev, V. P. Budaev, M. M. Dremin, E. V. Gerasimov, S. A. Grashin, L. N. Khimchenko, S. V. Krilov, Yu. D. Pavlov, D. A. Shelukhin, S. V. Soldatov, N. N. Timchenko, G. Van Oost, and V. A. Vershkov, Plasma Phys. Controlled Fusion 45, 337 (2003).

${ }^{26}$ S. Silva, H. Figueiredo, I. Nedzelsky, B. Goncalves, J. A. C. Cabral, C. A. F. Varandas, and G. Van Oost, Plasma Phys. Controlled Fusion 46, 163 (2004).

${ }^{27}$ L. Tramontin, L. Garzotti, V. Antoni, L. Carraro, D. Desideri, P. Innocente, E. Martines, G. Serianni, M. Spolaore, and N. Vianello, Plasma Phys. Controlled Fusion 44, 195 (2002).

${ }^{28}$ J. N. Talmadge, V. Sakaguchi, F. S. B. Anderson, D. T. Anderson, and A. F. Almagri, Phys. Plasmas 8, 5165 (2001).

${ }^{29}$ K. M. Likin, A. Abdou, A. F. Almagri, D. T. Anderson, F. S. B. Anderson, F. S. B. Anderson, D. Brower, J. Canik, C. Deng, S. P. Gerhardt, W. Guttenfelder, S. Oh, J. Radder, V. Sakaguchi, J. Schmitt, J. Tabora, J. N. Talmadge, and K. Zhai, Plasma Phys. Controlled Fusion 45, A133 (2003).

${ }^{30}$ K. C. Shaing and J. D. Callen, Phys. Fluids 26, 3315 (1983).

${ }^{31}$ M. Coronado and H. Wobig, Phys. Fluids 29, 527 (1986).

${ }^{32}$ P. S. Krstic and D. R. Schultz, At. Plasma-Mater. Interact. Data Fusion 8, 1 (1998).

${ }^{33}$ P. Bachmann and D. Reiter, Contrib. Plasma Phys. 35, 45 (1995).

${ }^{34}$ J. Cornelis, R. Sporken, G. Van Oost, and R. R. Weynants, Nucl. Fusion 34, 171 (1994).

${ }^{35}$ K. C. Shaing, S. P. Hirshman, and J. D. Callen, Phys. Fluids 29, 521 (1986).

${ }^{36}$ M. Coronado and H. Wobig, Phys. Fluids 29, 527 (1986).

${ }^{37}$ S. P. Hirshman, Nucl. Fusion 18, 917 (1978).

${ }^{38}$ G. Kuo-Petravic, A. H. Boozer, J. A. Rome, and R. H. Fowler, J. Comput. Phys. 51, 261 (1983).

${ }^{39}$ M. Coronado and J. G. Trejo, Phys. Fluids B 2, 530 (1990),

${ }^{40}$ K. C. Shaing, Phys. Fluids 29, 2231 (1986).
${ }^{41}$ S. P. Gerhardt, J. N. Talmandge, and D. T. Anderson, Phys. Plasmas 12, 012504 (2005).

${ }^{42}$ M. Coronado and H. Wobig, Phys. Fluids 30, 3171 (1987).

${ }^{43}$ H. Maassberg, C. D. Beidler, U. Gasparino, M. Rome, K. S. Dyabilin, N. B. Marushchenko, and S. Murakami, Phys. Plasmas 7, 295 (2000).

${ }^{44}$ W. M. Solomon and M. G. Shats, Phys. Rev. Lett. 87, 195003 (2001).

${ }^{45}$ L. G. Askinazi, V. E. Golant, S. V. Lebedev, V. A. Rozhanskij, and M. Tendler, Nucl. Fusion 32, 271 (1992).

${ }^{46}$ V. Rozhansky and M. Tendler, Phys. Fluids B 4, 1878 (1992).

${ }^{47}$ H. Weisen, M. Von Hellermann, A. Boileau, L. D. Horton, W. Mandl, and H. P. Summers, Nucl. Fusion 29, 2187 (1989).

${ }^{48}$ S. D. Scott, P. H. Diamond, R. J. Fonck, R. J. Goldston, R. B. Howell, K. P. Jaehnig, G. Schilling, E. J. Synakowski, M. C. Zarnstorff, C. E. Bush, E. Fredrickson, K. W. Hill, A. C. Janos, D. K. Mansfield, D. K. Owens, H. Park, G. Pautasso, A. T. Ramsey, J. Schivell, G. D. Tait, W. M. Tang, and G. Taylor, Phys. Rev. Lett. 64, 531 (1990).

${ }^{49}$ S. D. Scott, V. Arunasalan, C. W. Barnes, M. G. Bell, M. Bitter, R. Boivin, N. L. Bretz, R. Budny, C. E. Bush, A. Cavallo, T. K. Chu, S. A. Cohen, P. Colestock, S. L. Davis, D. L. Dimock, H. F. Dylla, P. C. Efthimion, A. B. Erhardt, R. J. Fonck, E. Fredrickson, H. P. Furth, R. J. Goldston, G. Greene, B. Grek, L. R. Grisham, G. Hammett, R. J. Hawryluk, H. W. Hendel, K. W. Hill, E. Hinnov, D. J. Hoffman, J. Hosea, R. B. Howell, H. Hsuan, R. A. Hulse, K. P. Jaehnig, A. C. Janos, D. Jassby, F. Jobes, D. W. Johnson, L. C. Johnson, R. Kaita, C. Kieras-Phillips, S. J. Kilpatrick, P. H. LaMarche, B. LeBlanc, R. Little, D. M. Manos, D. K. Mansfield, E. Mazzucato, M. P. McCarthy, D. C. McCune, K. McGuire, D. H. McNeill, D. M. Meade, S. S. Medley, D. R. Mikkelsen, R. Motley, D. Mueller, J. A. Murphy, Y. Nagayama, R. Nazakian, D. K. Owens, H. Park, A. T. Ramsey, M. H. Redi, A. L. Roquemore, P. H. Rutherford, G. Schilling, J. Schivell, G. L. Schmidt, J. Stevens, B. C. Stratton, W. Stodiek, E. J. Synakowski, W. M. Tang, G. Taylor, J. R. Timberlake, H. H. Towner, M. Ulrickson, S. von Goeler, R. Wieland, M. Williams, J. R. Wilson, K.-L. Wong, S. Yoshikawa, K. M. Young, M. C. Zarnstorff, and S. J. Zweben, Phys. Fluids B 2, 1300 (1990).

${ }^{50}$ A. Kallenbach, H. M. Mayer, G. Fussmann, V. Mertens, U. Stroth, and O. Vollmer, The ASDEX Team, Plasma Phys. Controlled Fusion 33, 595 (1991).

${ }^{51}$ J. S. deGrassie, D. R. Baker, K. H. Burrell, P. Gohil, C. M. Greenfield, R. J. Groebner, and D. M. Thomas, Nucl. Fusion 43, 142 (2003).

${ }^{52}$ W. D. Lee, J. E. Rice, E. A. Marmar, M. J. Greenwald, I. H. Hutchinson, and J. A. Snipes, Phys. Rev. Lett. 91, 205003 (2003). 